\title{
Discrete Zak Transforms, Polyphase Transforms, and Applications
}

\author{
Helmut Bölcskei, Student Member, IEEE, and Franz Hlawatsch, Member, IEEE
}

\begin{abstract}
We consider three different versions of the Zak transform (ZT) for discrete-time signals, namely, the discretetime ZT, the polyphase transform, and a cyclic discrete ZT. In particular, we show that the extension of the discrete-time ZT to the complex $z$-plane results in the polyphase transform, an important and well-known concept in multirate signal processing and filter bank theory.

We discuss fundamental properties, relations, and transform pairs of the three discrete $Z T$ versions, and we summarize applications of these transforms. In particular, the discrete-time $\mathrm{ZT}$ and the cyclic discrete $\mathrm{ZT}$ are important for discrete-time Gabor expansion (Weyl-Heisenberg frame) theory since they diagonalize the Weyl-Heisenberg frame operator for critical sampling and integer oversampling. The polyphase representation plays a fundamental role in the theory of filter banks, especially DFT filter banks. Simulation results are presented to demonstrate the application of the discrete ZT to the efficient calculation of dual Gabor windows, tight Gabor windows, and frame bounds.
\end{abstract}

\section{INTRODUCTION AND OUTLINE}

$\mathbf{T}$ HE ZAK TRANSFORM (ZT), which is also known as Weil-Brezin mapping, Gel'fand mapping, and $k q$ representation [1]-[5], plays an important role in the theory of Gabor expansions and Weyl-Heisenberg frames (WHF's) [6]-[24]. On the theoretical side, the ZT allows the formulation of theorems on the existence of WHF's. On the practical side, the ZT is an efficient tool for the numerical calculation of dual WHF's, tight WHF's, and frame bounds. The ZT is very useful in several other fields as well, such as the theory of coherent states [4], [5], wavelet transforms [25], linear equalizers [26], and filter bank theory [27]-[29].

A discrete-time [30]-[32], [28], [10], [19] and a cyclic, discrete-time/discrete-frequency ZT version [33], [34], [12], [16], [10] have recently been introduced. However, while an excellent tutorial paper on the continuous-time ZT exists [2], a similar work for the discrete ZT versions is lacking. The present paper, which is partly of survey nature but also presents original results, is an attempt to fill this gap. Specifically, we summarize and extend systematic discussions of some properties of discrete ZT's that have been provided by various authors [30], [32]-[35], [10], [12]. We also show that the discrete-time ZT extended to the complex $z$-plane [28] is the same as the polyphase transform important for multirate signal processing and filter banks [36]-[44], and we summarize the

Manuscript received May 11, 1996; revised October 14, 1996. This work was supported by FWF Grants P10012-ÖPH and P10531-ÖPH. The associate editor coordinating the review of this paper and approving it for publication was Dr. Jelena Kovacević.

The authors are with INTHFT, Vienna University of Technology, Gusshausstrasse 25/389, A-1040 Vienna, Austria.

Publisher Item Identifier S 1053-587X(97)02572-5. application of discrete ZT's to Gabor expansions (WHF's) and DFT filter banks.

The paper is organized as follows. In Section II, we define the discrete-time ZT (DTZT), which is discrete in time but continuous and periodic in frequency, and we discuss basic properties of the DTZT. Section III considers the application of the DTZT to the discrete-time Gabor expansion. We review the Zibulski-Zeevi method for calculating dual WHF's, and we show that the DTZT diagonalizes the Gabor (Weyl-Heisenberg) frame operator for critical sampling and integer oversampling, which makes the DTZT-based calculation of dual and tight Gabor frames and frame bounds very efficient. We furthermore find the (generalized) eigenfunctions and eigenvalues of the Gabor frame operator for critical sampling and integer oversampling.

In Section IV, the DTZT is extended to the complex $z$ plane, which is shown to result in the well-known polyphase transform (PT). Important properties and transform pairs of the PT are provided. Section V reviews the application of the PT to oversampled and critically sampled DFT filter banks. Although some of the observations of Section V hold for more general filter banks [27], [29], we shall restrict our attention to DFT filter banks for the sake of coherence with other sections.

Section VI introduces the discrete ZT (DZT), which is discrete in time and frequency and, thus, particularly appropriate for numerical implementations. Important properties of the DZT are discussed. In Section VII, we show that the DZT allows an efficient, FFT-based implementation [33], [12] of a cyclic version of the discrete Gabor expansion [8] for critical sampling and integer oversampling. Finally, Section VIII presents simulation results demonstrating the importance of the DZT for the efficient calculation of dual and tight Gabor windows as well as frame bounds.

\section{ThE Discrete-TIME ZAK TRANSFORM}

In this section, we consider the discrete-time ZT (DTZT) [30]-[32], [28], [10], [19] and discuss its properties. Most of these properties are obtained by straightforward discretization of results found for the continuous-time case by Janssen [2].

1) Definition and Interpretation: The DTZT can be defined by sampling the continuous-time ZT with respect to time. The continuous-time ZT of a signal $x(t)$ is defined as [2]

$$
Z_{x}^{(T)}(t, f)=\sqrt{T} \sum_{l=-\infty}^{\infty} x(t+l T) e^{-j 2 \pi l T f}
$$

where $t$ is time, $f$ is frequency, and $T>0$ is fixed time period parameter. Evaluating the ZT at uniformly spaced time instants 
$t=n T / L$, where $L \in \mathbb{N}$, setting $f=\theta / T$, and dividing by $\sqrt{T}$, we obtain

$$
\begin{aligned}
Z_{x}^{(L)}(n, \theta) & \triangleq \frac{1}{\sqrt{T}} Z_{x}^{(T)}\left(n \frac{T}{L}, \frac{\theta}{T}\right) \\
& =\sum_{l=-\infty}^{\infty} x\left(n \frac{T}{L}+l T\right) e^{-j 2 \pi l \theta}
\end{aligned}
$$

Introducing the discrete-time signal $x[n] \triangleq x(n T / L)$ as a sampled version of $x(t)$ with sampling period $T / L$, the DTZT is finally formulated as

$$
Z_{x}^{(L)}(n, \theta)=\sum_{l=-\infty}^{\infty} x[n+l L] e^{-j 2 \pi l \theta}
$$

The convergence of the series (1) is discussed in [30]. The DTZT is a linear signal transformation that maps a discretetime signal $x[n]$ onto a 2-D function of the discrete time index $n$ and the (continuous) normalized frequency variable $\theta$. The DTZT parameter $L$ is analogous to the parameter $T$ in the continuous-time ZT. We shall usually omit the superscript $(L)$ in order to simplify the notation. In what follows, we assume that $x[n] \in l^{2}(\mathbb{Z})$, where $l^{2}(\mathbb{Z})$ is the space of square-summable (finite-energy) discrete-time signals with inner product $\langle x, y\rangle=\sum_{n=-\infty}^{\infty} x[n] y^{*}[n]$ and norm $\|x\|=$ $\sqrt{\langle x, x\rangle}=\sqrt{\sum_{n=-\infty}^{\infty}|x[n]|^{2}}$.

The DTZT at a fixed time index $n=n_{0}$ is the discrete-time Fourier transform of the signal $x_{n_{0}}[l]=x\left[n_{0}+l L\right]$ derived by subsampling $x\left[n_{0}+n\right]$ by a factor of $L$

$$
Z_{x}\left(n_{0}, \theta\right)=\sum_{l=-\infty}^{\infty} x_{n_{0}}[l] e^{-j 2 \pi l \theta}
$$

In particular, the DTZT for $L=1$ evaluated at $n=0$ is the discrete-time Fourier transform

$$
Z_{x}^{(1)}(0, \theta)=X(\theta)=\sum_{l=-\infty}^{\infty} x[l] e^{-j 2 \pi l \theta}
$$

2) Frequency-Domain Expression: The DTZT can be expressed in terms of $X(\theta)$ (see above) as

$$
Z_{x}(n, \theta)=\frac{1}{L} e^{j 2 \pi \theta n / L} \sum_{m=0}^{L-1} X\left(\frac{\theta+m}{L}\right) e^{j 2 \pi(m / L) n}
$$

In the remainder of this section, we shall discuss important DTZT properties and relations, such as periodicity properties, relations involving integrals and/or sums of the DTZT, and shift properties. We shall show that the DTZT is a unitary mapping, i.e., a one-to-one mapping that preserves norms and inner products. We shall furthermore discuss situations where the symmetry of a signal implies that its DTZT has one or more zeros. (The zeros of the DTZT play a fundamental role in WHF theory; see Section III-B.)
3) Periodicity: The DTZT is quasiperiodic in $n$ and periodic in $\theta$ :

$$
\begin{aligned}
Z_{x}(n+l L, \theta) & =e^{j 2 \pi l \theta} Z_{x}(n, \theta), \\
Z_{x}(n, \theta+l) & =Z_{x}(n, \theta) \quad \text { with } l \in \mathbb{Z} .
\end{aligned}
$$

Hence, it suffices to know the DTZT on the "fundamental rectangle" $(n, \theta) \in[0, L-1] \times[0,1)$.

4) Integral/Sum Relations: Some important relations involve integrals and/or sums of the DTZT. First, the signal $x[n]$ can be recovered from its DTZT by integrating the DTZT with respect to frequency $\theta$ :

$$
\int_{0}^{1} Z_{x}(n, \theta) d \theta=x[n]
$$

We can also recover the signal's Fourier transform from $Z_{x}(n, \theta)$ as

$$
\sum_{n=0}^{L-1} Z_{x}(n, \theta L) e^{-j 2 \pi n \theta}=X(\theta)
$$

This is related to the polyphase decomposition in multirate signal processing [36]-[44] (see Sections IV and V).

Besides being invertible, the DTZT also preserves inner products and norms:

$$
\left\langle Z_{x}, Z_{y}\right\rangle=\langle x, y\rangle \quad \text { and } \quad\left\|Z_{x}\right\|=\|x\|
$$

where the inner product and norm in the DTZT domain are defined as

$$
\begin{aligned}
\left\langle Z_{x}, Z_{y}\right\rangle & =\sum_{n=0}^{L-1} \int_{0}^{1} Z_{x}(n, \theta) Z_{y}^{*}(n, \theta) d \theta, \\
\left\|Z_{x}\right\| & =\sqrt{\left\langle Z_{x}, Z_{x}\right\rangle}=\sqrt{\sum_{n=0}^{L-1} \int_{0}^{1}\left|Z_{x}(n, \theta)\right|^{2} d \theta .}
\end{aligned}
$$

Two further sum/integral relations are

$$
\begin{aligned}
& \sum_{n=0}^{L-1} Z_{x}(n, \theta) Z_{y}^{*}(n, \theta)=\frac{1}{L} \sum_{m=0}^{L-1} X\left(\frac{\theta+m}{L}\right) Y^{*}\left(\frac{\theta+m}{L}\right) \\
& \int_{0}^{1} Z_{x}(n, \theta) Z_{y}^{*}(n, \theta) d \theta=\sum_{l=-\infty}^{\infty} x[n+l L] y^{*}[n+l L] .
\end{aligned}
$$

5) Unitarity: The DTZT is a unitary mapping from $l^{2}(\mathbb{Z})$ onto $l^{2}([0, L-1] \times[0,1))$, which is the space of functions $F(n, \theta)$ defined for $n \in[0, L-1]$ and $\theta \in[0,1)$ and satisfying

$$
\|F\|=\sqrt{\sum_{n=0}^{L-1} \int_{0}^{1}|F(n, \theta)|^{2} d \theta}<\infty .
$$

The unitarity of the DTZT has two important consequences:

- The DTZT is a one-to-one mapping. In particular, to any $F(n, \theta) \in l^{2}([0, L-1] \times[0,1))$, we can find a unique signal $x[n] \in l^{2}(\mathbb{Z})$ such that $Z_{x}(n, \theta)=F(n, \theta)$. This signal can be obtained according to the inversion formula (3) as $x[n]=\int_{0}^{1} F(n, \theta) d \theta$.

- The DTZT preserves inner products and norms [see (5)]. 
TABLE I

Signal Transformation Properties of the DTZT

\begin{tabular}{|l|l||l|l|}
\hline Signal & DTZT & Signal & DTZT \\
\hline$a x[n]+b y[n]$ & $a Z_{x}(n, \theta)+b Z_{y}(n, \theta)$ & $x[n] * y[n]$ & $\sum_{m=0}^{L-1} Z_{x}(n-m, \theta) Z_{y}(m, \theta)$ \\
$x[-n]$ & $Z_{x}(-n,-\theta)$ & $x[n K]$ & $Z_{x}^{(K L)}(n K, \theta)$ \\
$x^{*}[n]$ & $Z_{x}^{*}(n,-\theta)$ & $x[n-N]$ & $Z_{x}(n-N, \theta)$ \\
$x^{*}[-n]$ & $Z_{x}^{*}(-n, \theta)$ & $x[n] e^{j 2 \pi \theta_{0} n}$ & $e^{j 2 \pi \theta_{0} n} Z_{x}\left(n, \theta-L \theta_{0}\right)$ \\
$\operatorname{Re}\{x[n]\}$ & $\frac{Z_{x}(n, \theta)+Z_{x}^{*}(n,-\theta)}{2}$ & $x[n-l L]$ & $e^{-j 2 \pi l \theta} Z_{x}(n, \theta)$ \\
$\operatorname{Im}\{x[n]\}$ & $\frac{Z_{x}(n, \theta)-Z_{x}^{*}(n,-\theta)}{2 j}$ & $x[n-l L] e^{j 2 \pi \frac{m}{M} n}$ & $e^{-j 2 \pi l\left(\theta-\frac{m}{M} L\right)} e^{j 2 \pi \frac{m}{M} n} Z_{x}\left(n, \theta-\frac{m}{M} L\right)$ \\
$x_{e}[n]=\frac{x[n]+x^{*}[-n]}{2}$ & $\frac{Z_{x}(n, \theta)+Z_{x}^{*}(-n, \theta)}{2}$ & $n[n-l L] e^{j 2 \pi \frac{m}{L} n}$ & $e^{-j 2 \pi l \theta} e^{j 2 \pi \frac{m}{L} n} Z_{x}(n, \theta)$ \\
$x_{0}[n]=\frac{x[n]-x^{*}[-n]}{2}$ & $\frac{Z_{x}(n, \theta)-Z_{x}^{*}(-n, \theta)}{2}$ & $\sum_{k=0}^{l} x[n]$ & $\left.n n^{l}\right) n^{k} L^{l-k}\left(-\frac{1}{2 \pi j}\right)^{l-k} \frac{\partial^{l-k} Z_{x}(n, \theta)}{\partial^{l-k} \theta}$ \\
$x[n] y[n]$ & $\int_{0}^{1} Z_{x}(n, \theta-\zeta) Z_{y}(n, \zeta) d \zeta$ & $n x[n]$ & $n Z_{x}(n, \theta)-\frac{L}{2 \pi j} \frac{\partial Z_{x}(n, \theta)}{\partial \theta}$ \\
\hline
\end{tabular}

Proof of Unitarity: Following [45, pp. 109-110], it suffices to show that the DTZT maps an orthonormal basis of $l^{2}(\mathbb{Z})$ to an orthonormal basis of $l^{2}([0, L-1] \times[0,1))$. Let $g[n]=1$ for $0 \leq n \leq L-1$ and $g[n]=0$ otherwise. The DTZT of $g[n]$ is $Z_{g}(n, \theta)=1$ on the fundamental rectangle. The set of signals $g_{l, m}[n]=\frac{1}{\sqrt{L}} g[n-l L] e^{j 2 \pi(m / L) n}$ with $l \in \mathbb{Z}$ and $m \in[0, L-1]$ is easily shown to constitute an orthonormal basis for $l^{2}(\mathbb{Z})$. The DTZT of $g_{l, m}[n]$ is given by $Z_{g_{l, m}}(n, \theta)=$ $\frac{1}{\sqrt{L}} e^{-j 2 \pi l \theta} e^{j 2 \pi(m / L) n} Z_{g}(n, \theta)=\frac{1}{\sqrt{L}} e^{-j 2 \pi l \theta} e^{j 2 \pi(m / L) n}$. It is easily checked that the functions $Z_{g_{l, m}}(n, \theta)$ constitute an orthonormal basis for $l^{2}([0, L-1] \times[0,1))$.

6) Product of Two DTZT's: With (2), the DTZT product $Z_{x}(n, \theta) Z_{y}^{*}(n, \theta)$ is $L$-periodic with respect to time $n$ and 1-periodic with respect to frequency $\theta$. Therefore, $Z_{x}(n, \theta) Z_{y}^{*}(n, \theta)$ can be represented by a 2 -D Fourier series expansion, which can be shown to read as follows:

$$
Z_{x}(n, \theta) Z_{y}^{*}(n, \theta)=\frac{1}{L} \sum_{l=-\infty}^{\infty} \sum_{m=0}^{L-1}\left\langle x, y_{l, m}\right\rangle e^{j 2 \pi(m / L) n} e^{-j 2 \pi l \theta}
$$

with $y_{l, m}[n]=y[n-l L] e^{j 2 \pi(m / L) n}$. We note that the Fourier series coefficients

$$
\left\langle x, y_{l, m}\right\rangle=\sum_{n=-\infty}^{\infty} x[n] y^{*}[n-l L] e^{-j 2 \pi(m / L) n}
$$

are samples of the discrete-time short-time Fourier transform of $x[n]$ using the analysis window $y[n]$ [46], [47] (or, equivalently, samples of the discrete cross-ambiguity function of $x[n]$ and $y[n][33])$. By inversion of (6), we obtain

$$
\left\langle x, y_{l, m}\right\rangle=\sum_{n=0}^{L-1} \int_{0}^{1} Z_{x}(n, \theta) Z_{y}^{*}(n, \theta) e^{-j 2 \pi(m / L) n} e^{j 2 \pi l \theta} d \theta .
$$

7) Zeros of the DTZT: When $L$ is even and $x[n]$ is even, i.e., $x[-n]=x[n]$, then the DTZT has a zero in the center of the fundamental rectangle, $Z_{x}(L / 2,1 / 2)=0$. When $L$ is even and $x[n]$ is odd, i.e., $x[-n]=-x[n]$, then $Z_{x}(L / 2,0)=0$.
For $x[n]$ odd and $L$ even or odd, we have $Z_{x}(0,0)=$ $0, Z_{x}(0,1 / 2)=0$. The significance of the zeros of the DTZT in the context of WHF theory will be discussed in Section III-B.

8) Time-Limited Signals: If $x[n]$ is zero outside the interval $[0, L-1]$, then

$$
Z_{x}(n, \theta)=x[n] \text { for } 0 \leq n \leq L-1 .
$$

If $x[n]$ is zero outside the interval $[0, K L-1]$, then

$$
\frac{1}{K} \sum_{k=0}^{K-1} Z_{x}\left(n, \frac{k}{K}\right)=x[n] \quad \text { for } \quad 0 \leq n \leq K L-1 .
$$

This formula can be considered to be a discretized version of (3).

9) Bandlimited Signals: If $x[n]$ is bandlimited to the frequency band $[0,1 / L)$, i.e., $X(\theta)=0$ for $1 / L \leq \theta<1$, then

$$
Z_{x}(n, \theta)=\frac{1}{L} e^{j 2 \pi \theta n / L} X\left(\frac{\theta}{L}\right) .
$$

If $x[n]$ is bandlimited in an interval $[0, K / L)$, where $L / K \in$ $\mathbb{N}$, then

$$
\frac{L}{K} \sum_{k=0}^{K-1} Z_{x}\left(k \frac{L}{K}, L \theta\right) e^{-j 2 \pi \theta k L / K}=X(\theta) \text { for } 0 \leq \theta<\frac{K}{L} .
$$

This is a discretized version of (4).

10) Signal Transformation Properties, Symmetry Relations, and Transform Pairs: Table I lists further important DTZT relations that show how the DTZT behaves under various signal transformations. Table II summarizes symmetry relations of the DTZT, and Table III provides some DTZT transform pairs. In these tables, we have $x[n] * y[n]=\sum_{m=-\infty}^{\infty} x[n-m] y[m]$; furthermore, $u[n]$ is the unit step function, $\delta[n]$ is the discretetime delta function (or unit sample), $\delta(\theta)$ is the Dirac delta function, and $\operatorname{sinc}(\alpha)=\sin \alpha / \alpha$. 
TABLE II

SYMMETRY RELATIONS OF THE DTZT

\begin{tabular}{|l|l|l|l|}
\hline Signal property & DTZT property & Signal property & DTZT property \\
\hline$x[-n]=x[n]$ & $Z_{x}(-n,-\theta)=Z_{x}(n, \theta)$ & $x^{*}[n]=x[n]$ & $Z_{x}^{*}(n,-\theta)=Z_{x}(n, \theta)$ \\
$x^{*}[-n]=x[n]$ & $Z_{x}^{*}(-n, \theta)=Z_{x}(n, \theta)$ & $x^{*}[n]=-x[n]$ & $Z_{x}^{*}(n,-\theta)=-Z_{x}(n, \theta)$ \\
\hline
\end{tabular}

TABLE III

DTZT Transform Pairs. (The DTZT Expressions Are Valid for $(n, \theta) \in[0, L-1] \times[0,1)$.)

\begin{tabular}{|l|l||l|l|}
\hline$x[n]$ & $Z_{x}(n, \theta)$ & $x[n]$ & $Z_{x}(n, \theta)$ \\
\hline$\delta\left[n-n_{0}\right]$ & $\delta\left[n-n_{0}\right], \quad 0 \leq n_{0} \leq L-1$ & $e^{j 2 \pi \theta_{0} n}$ & $e^{j 2 \pi \theta_{0} n} \delta\left(\theta-L \theta_{0}\right)$ \\
$u[n]$ & $\frac{1}{1-e^{-j 2 \pi \theta}}$ & $u[n]-u[n-L]$ & 1 \\
1 & $\delta(\theta)$ & $a^{n} u[n], \quad|a|<1$ & $\frac{a^{n} e^{j 2 \pi \theta}}{e^{j 2 \pi \theta}-a^{L}}$ \\
$e^{j \pi \frac{n}{L} \operatorname{sinc}\left(\frac{\pi n}{L}\right)}$ & $e^{j 2 \pi \theta^{n}}$ & $a^{|n|}, \quad|a|<1$ & $\frac{a^{n} e^{j 2 \pi \theta}}{e^{j 2 \pi \theta}-a^{L}}+\frac{a^{-n} e^{-j 2 \pi \theta}}{e^{-j 2 \pi \theta}-a^{L}}-\delta[n]$ \\
\hline
\end{tabular}

\section{DISCRETE-TIME GABOR EXPANSIONS}

The discrete-time Gabor expansion is an important timefrequency signal expansion that has been studied, e.g., in [8], [10], [12], [33], [48], and [20] and extended to the multiwindow case in [16]. This section discusses the application of the DTZT to the discrete-time Gabor expansion [30], [19], [31]. After a review of the Zibulski-Zeevi method for calculating dual frames [12], we show that the DTZT diagonalizes the Gabor (or Weyl-Heisenberg) frame operator in the cases of critical sampling and integer oversampling and thereby allows a simple calculation of the dual Gabor window, the frame bounds, and tight Gabor windows [12], [16], [31].

The discrete-time Gabor expansion of a signal $x[n] \in l^{2}(\mathbb{Z})$ is defined as

$$
x[n]=\sum_{l=-\infty}^{\infty} \sum_{m=0}^{M-1} a_{l, m} g_{l, m}[n]
$$

with $g_{l, m}[n]=g[n-l L] e^{j 2 \pi(m / M) n}$, where $a_{l, m}$ are the Gabor coefficients, $g[n]$ is a suitably chosen "synthesis window," and $L, M \in \mathbb{N}$ with $M \geq L$ are the grid parameters. The Gabor coefficients can be calculated as

$$
a_{l, m}=\left\langle x, \gamma_{l, m}\right\rangle=\sum_{n=-\infty}^{\infty} x[n] \gamma_{l, m}^{*}[n]
$$

where $\gamma_{l, m}[n]=\gamma[n-l L] e^{j 2 \pi(m / M) n}$ with a suitable "analysis window" $\gamma[n]$. In the cases of oversampling $(M>L)$ and critical sampling $(M=L)$, the Gabor expansion (7), (8) exists for arbitrary $x[n] \in l^{2}(\mathbb{Z})$ if the windows $g[n]$ and $\gamma[n]$ are chosen properly (see below). In the case of undersampling $(M<L)$, the set $\left\{g_{l, m}[n]\right\}$ is incomplete in $l^{2}(\mathbb{Z})$, and hence, it is not possible to expand every signal $x[n] \in l^{2}(\mathbb{Z})$ into the $g_{l, m}[n]$. (It is well known that this observation also holds for more general signal expansions; see, for example, [27] and [29].)

\section{A. Discrete-Time Weyl-Heisenberg Frames}

The theory of Weyl-Heisenberg frames (WHF's) [45], [49], [50] yields important results on the Gabor expansion. For $M \geq L$, a set of functions $g_{l, m}[n]=g[n-l L] e^{j 2 \pi(m / M) n}$ with $-\infty<l<\infty$ and $0 \leq m \leq M-1$ is said to be a WHF for $l^{2}(\mathbb{Z})$ if for all $x[n] \bar{\epsilon} l^{2}(\overline{\mathbb{Z}})$

$$
\begin{aligned}
& A\|x\|^{2} \leq \sum_{l=-\infty}^{\infty} \sum_{m=0}^{M-1}\left|\left\langle x, g_{l, m}\right\rangle\right|^{2} \leq B\|x\|^{2} \\
& \text { with } 0<A \leq B<\infty \text {. }
\end{aligned}
$$

The constants $A>0$ and $B<\infty$ are called frame bounds. For the continuous-time case, analytic expressions for the frame bounds of some specific window functions can be found in [51]. For synthesis window $g[n]$ such that $\left\{g_{l, m}[n]\right\}$ is a WHF, the Gabor expansion (7), (8) exists for all $x[n] \in l^{2}(\mathbb{Z})$, and an analysis window (or "dual" window) can be derived from $g[n]$ as

$$
\gamma[n]=\left(S^{-1} g\right)[n]
$$

Here, $S^{-1}$ is the inverse of the frame operator $S$ defined as

$$
(\boldsymbol{S} x)[n]=\sum_{l=-\infty}^{\infty} \sum_{m=0}^{M-1}\left\langle x, g_{l, m}\right\rangle g_{l, m}[n] .
$$

The frame operator is a linear, positive definite operator (corresponding to a positive definite matrix of infinite size) mapping $l^{2}(\mathbb{Z})$ onto $l^{2}(\mathbb{Z})$. If $\left\{g_{l, m}[n]\right\}$ is a WHF with frame bounds $A$ and $B$, then $\left\{\gamma_{l, m}[n]\right\}$ is a WHF as well (the "dual" frame), with frame bounds $\tilde{A}=1 / B$ and $\tilde{B}=1 / A$ [45], [49], [50]. The numerical properties of the Gabor expansion will be better for closer frame bounds $A$ and $B$. A WHF is called snug if $A \approx B$ and tight if $A=B$. For a tight WHF, $S=A \boldsymbol{I}$, where $I$ is the identity operator on $l^{2}(\mathbb{Z})$, and hence, there is simply $\gamma[n]=(1 / A) g[n]$. From a synthesis window $g[n]$ generating a WHF, we can derive a "tight" synthesis window $g_{T}[n]$ (i.e., a 
window generating a tight WHF with frame bound $A=1$ ) as

$$
g_{T}[n]=\left(S^{-1 / 2} g\right)[n]
$$

where $S^{-1 / 2}$ is the inverse of the positive definite operator square root of $S$.

A WHF $\left\{g_{l, m}[n]\right\}$ with critical sampling is an exact frame, i.e., the $g_{l, m}[n]$ are linearly independent [45], [49], [50]. Here, both the analysis window $\gamma[n]$ and the Gabor coefficients $a_{l, m}$ are uniquely determined.

\section{B. Zibulski-Zeevi Method and Diagonalization of the Gabor Frame Operator}

We shall now briefly review the Zibulski-Zeevi method for the calculation of dual frames [12]. This method is based on the observation that the Gabor frame operator $\boldsymbol{S}$ can be expressed in the DTZT domain as

$$
\begin{aligned}
Z_{\boldsymbol{S}_{x}}(n, \theta)= & \frac{L}{Q} \sum_{q=0}^{Q-1} Z_{x}\left(n-q \frac{P}{Q} L, \theta\right) \\
& \cdot \sum_{p=0}^{P-1} Z_{g}\left(n, \theta-\frac{p}{P}\right) Z_{g}^{*}\left(n-q \frac{P}{Q} L, \theta-\frac{p}{P}\right)
\end{aligned}
$$

where $M=(P / Q) L$ with $P / Q \geq 1$. Based on (12), it can be shown that the inversion of the frame operator (i.e., the calculation of the dual frame) reduces to the inversion of a matrix of finite size. This method can be applied in the cases of rational oversampling $(M=(P / Q) L)$ and integer oversampling $(M=P L)$. For integer oversampling, including critical sampling as a special case, (12) simplifies to

$$
\begin{aligned}
Z_{\boldsymbol{S}_{x}}(n, \theta) & =\lambda_{g}(n, \theta) Z_{x}(n, \theta) \quad \text { with } \\
\lambda_{g}(n, \theta) & =L \sum_{p=0}^{P-1}\left|Z_{g}\left(n, \theta-\frac{p}{P}\right)\right|^{2} .
\end{aligned}
$$

Hence, the frame operator becomes a simple multiplication operator in the DTZT domain, i.e., the DTZT "diagonalizes" the Gabor frame operator in the case of integer oversampling.

This diagonalization can be interpreted in terms of the (generalized) eigenvalues $\lambda$ and (generalized) eigenfunctions $u[n]$ of the Gabor frame operator $S$, which are defined by $^{1}(\boldsymbol{S} u)[n]=\lambda u[n]$ [53]. For critical sampling or integer oversampling $(M=P L$ with $P \in \mathbb{N}$ ), we show in the Appendix that a set of generalized eigenfunctions of $S$ that is orthogonal and complete (in a generalized sense [53]) is

$$
\begin{aligned}
u_{n, \theta}\left[n^{\prime}\right] & =v\left[n^{\prime}-n\right] e^{j 2 \pi(\theta / L)\left(n^{\prime}-n\right)} \quad \text { with } \\
v[n] & =\sum_{l=-\infty}^{\infty} \delta[n-l L]
\end{aligned}
$$

(this result has previously been stated without proof in [31]). Note that the eigenfunctions are parameterized by time $n$

\footnotetext{
${ }^{1}$ The operator $S$ is not compact and has a continuous spectrum [52]. The $u[n]$ are not in $l^{2}(\mathbb{Z})$ and are hence called generalized eigenfunctions. A rigorous treatment of generalized eigenexpansions of such operators requires the theory of Gel'fand triplets of spaces [53] and is beyond the scope of this paper.
}

and normalized frequency $\theta$ (the continuous index $\theta$ reflects that the spectrum of $\boldsymbol{S}$ is continuous). The eigenfunctions are related to the DTZT as ${ }^{2}$

$$
Z_{x}(n, \theta)=\left\langle x, u_{n, \theta}\right\rangle
$$

where $x[n] \in l^{2}(\mathbb{Z})$. The eigenvalues are related to the DTZT as $\lambda_{n, \theta}=L \sum_{p=0}^{P-1}\left|Z_{g}\left(n, \theta-\frac{p}{P}\right)\right|^{2}$ (see the Appendix). We see that the DTZT of the Gabor synthesis window $g[n]$ determines the eigenvalues of the Gabor frame operator. The dependence of $\lambda_{n, \theta}$ on $g[n]$ will be emphasized by writing the eigenvalues as $\lambda_{g}(n, \theta)$ [see (13)]. For critical sampling $(P=1)$, (13) simplifies to $\lambda_{g}(n, \theta)=L\left|Z_{g}(n, \theta)\right|^{2}$.

We note that a second complete and orthogonal set of eigenfunctions of $S$ is $\bar{u}_{n, \theta}\left[n^{\prime}\right]=\bar{v}\left[n^{\prime}-n\right] e^{j 2 \pi(\theta / L)\left(n^{\prime}-n\right)}$ with $\bar{v}[n]=\sqrt{P} \sum_{m=-\infty}^{\infty} \delta[n-m M]$. These eigenfunctions lead to the "dual DTZT"

$$
\bar{Z}_{x}(n, \theta) \triangleq\left\langle x, \bar{u}_{n, \theta}\right\rangle=\sqrt{P} \sum_{m=-\infty}^{\infty} x[n+m M] e^{-j 2 \pi m P \theta}
$$

whose fundamental rectangle is $(n, \theta) \in[0, M-1] \times[0,1 / P)$. The dual DTZT satisfies $\bar{Z}_{x}(n+l M, \theta)=e^{j 2 \pi l P \theta} \bar{Z}_{x}(n, \theta)$ and $\bar{Z}_{x}\left(n, \theta+\frac{l}{P}\right)=\bar{Z}_{x}(n, \theta)$ with $l \in \mathbb{Z}$. Similarly to (13), we get

$$
\begin{aligned}
\bar{Z}_{S_{x}}(n, \theta) & =\bar{\lambda}_{g}(n, \theta) \bar{Z}_{x}(n, \theta) \quad \text { with } \\
\bar{\lambda}_{g}(n, \theta) & =M \sum_{p=0}^{P-1}\left|\bar{Z}_{g}(n+p L, \theta)\right|^{2} .
\end{aligned}
$$

It can furthermore be shown that $\bar{\lambda}_{g}(n, \theta)=\lambda_{g}(n, \theta)$. This interesting duality relation has also been observed in [12] and [54]. For critical sampling $(P=1), \bar{v}[n]=v[n]$ and $\bar{Z}_{x}(n, \theta)=Z_{x}(n, \theta)$.

The importance of the DTZT for the Gabor expansion is based on the above relations of the DTZT with the eigenvalues and eigenfunctions of the Gabor frame operator. Taking the inner product of some signal $x[n] \in l^{2}(\mathbb{Z})$ with both sides of the eigenequation $\left(S u_{n, \theta}\right)\left[n^{\prime}\right]=\lambda_{g}(n, \theta) u_{n, \theta}\left[n^{\prime}\right]$, we obtain $\left\langle x, \boldsymbol{S} u_{n, \theta}\right\rangle=\lambda_{g}(n, \theta)\left\langle x, u_{n, \theta}\right\rangle$, and further, $\left\langle\boldsymbol{S} x, u_{n, \theta}\right\rangle=$ $\lambda_{g}(n, \theta)\left\langle x, u_{n, \theta}\right\rangle$, where we have used $S^{*}=S$ (i.e., $S$ is self-adjoint) with $\boldsymbol{S}^{*}$ denoting the adjoint of $\boldsymbol{S}$ [52]. Thus, we have rederived (13) using (15).

We shall now summarize important consequences of the diagonalization discussed above [12].

- The inverse frame operator $S^{-1}$ can be expressed in the DTZT domain as a pointwise division

$$
Z_{S^{-1} x}(n, \theta)=\frac{Z_{x}(n, \theta)}{\lambda_{g}(n, \theta)} .
$$

- The frame condition (9) can be reformulated, using the DTZT of the synthesis window $g[n]$, as

$$
A \leq \lambda_{g}(n, \theta) \leq B \quad \text { with } \quad 0<A \leq B<\infty .
$$

In addition, the frame bounds $A, B$ are given by the infimum and the supremum, respectively, of $\lambda_{g}(n, \theta)$. In

\footnotetext{
${ }^{2}$ Strictly speaking, this inner product is not well defined since the $u_{n, \theta}\left[n^{\prime}\right]$ are not in $l^{2}(\mathbb{Z})$. It must be interpreted as the linear functional assigning to the signal $x[n]$ the value of its DTZT at $n, \theta$.
} 
particular, $\left\{g_{l, m}[n]\right\}$ is a tight WHF with frame bound $A$ if and only if $\lambda_{g}(n, \theta) \equiv A$.

- With (16), the analysis window $\gamma[n]$ in (10) can be calculated via the DTZT using

$$
Z_{\gamma}(n, \theta)=\frac{Z_{g}(n, \theta)}{\lambda_{g}(n, \theta)}
$$

and deriving $\gamma[n]$ according to (3), i.e., $\gamma[n]=$ $\int_{0}^{1} Z_{\gamma}(n, \theta) d \theta$.

- From a synthesis window $g[n]$ generating a WHF, a "tight" synthesis window $g_{T}[n]$ generating a tight WHF with frame bound $A=1$ can be derived using [cf. (11)]

$$
Z_{g_{T}}(n, \theta)=\frac{Z_{g}(n, \theta)}{\sqrt{\lambda_{g}(n, \theta)}}
$$

Equations analogous to (16)-(18) have first been reported by Daubechies et al. in [49] and by Zibulski and Zeevi in [12]. We furthermore note that the frame operator of the dual frame $\left\{\gamma_{l, m}[n]\right\}$ is $\boldsymbol{S}^{-1}$ with eigenvalues $\lambda_{\gamma}(n, \theta)=$ $L \sum_{p=0}^{P-1}\left|Z_{\gamma}\left(n, \theta-\frac{p}{P}\right)\right|^{2}=1 / \lambda_{g}(n, \theta)$ (assuming appropriate ordering of the eigenvalues). Hence, the DTZT of the synthesis window $g[n]$ can be obtained from the DTZT of the analysis window $\gamma[n]$ as $Z_{g}(n, \theta)=Z_{\gamma}(n, \theta) / \lambda_{\gamma}(n, \theta)$.

In the case of critical sampling $(P=1)$, the above relations simplify to

$$
\begin{aligned}
A & \leq L\left|Z_{g}(n, \theta)\right|^{2} \leq B, \quad Z_{\gamma}(n, \theta)=\frac{1}{L Z_{g}^{*}(n, \theta)} \\
Z_{g_{T}}(n, \theta) & =\frac{1}{\sqrt{L}} e^{j \arg \left\{Z_{g}(n, \theta)\right\}}
\end{aligned}
$$

with $A>0$. The DTZT frame condition $A \leq L\left|Z_{g}(n, \theta)\right|^{2} \leq$ $B$ has an interesting implication. In the continuous-time case, the Balian-Low theorem [45] states that only synthesis windows that are either not very smooth or have poor decay generate a critically sampled WHF; this, in particular, excludes the Gaussian window [49]. In the discrete-time case, i.e., for WHF's in $l^{2}(\mathbb{Z})$, Heil [30] has shown that the situation is different. For $L$ even, the DTZT of the discrete-time (sampled) Gaussian has a zero; therefore, $0<A \leq L\left|Z_{g}(n, \theta)\right|^{2}$ in (20) is not satisfied, and the corresponding Weyl-Heisenberg set $\left\{g_{l, m}[n]\right\}$ is not a WHF for $l^{2}(\mathbb{Z})$. However, for $L$ odd, the DTZT of the sampled Gaussian does not have a zero, and therefore, $\left\{g_{l, m}[n]\right\}$ is a WHF for $l^{2}(\mathbb{Z})$ (even though $A$ must still be expected to be close to 0 ). This shows that the Balian-Low theorem does not carry over to the discrete-time case in a straightforward manner.

\section{The Polyphase Transform}

The polyphase decomposition introduced in [36] is of fundamental importance in filter bank theory. In [37]-[44], the application of the polyphase decomposition to the analysis and design of filter banks is discussed. In this and the next section, we shall show that the extension of the DTZT to the $z$-plane yields the transform underlying the polyphase decomposition. Fundamental properties of this transform will be studied, thereby establishing known and new results on the polyphase decomposition. The application of the polyphase transform to DFT filter banks with critical sampling or integer oversampling is discussed in Section V.

1) Definition and Interpretation: In analogy to the $z$-transform, which is the extension of the discrete-time Fourier transform to the complex $z$-plane [55], we define the polyphase transform (PT) by extending the DTZT in (1) to the $z$-plane. Formally replacing $e^{j 2 \pi \theta}$ by $z$, we obtain

$$
\mathcal{Z}_{x}^{(L)}(n, z) \triangleq \sum_{l=-\infty}^{\infty} x[n+l L] z^{-l}
$$

We use a script $\mathcal{Z}$ to distinguish the PT from the DTZT. We shall usually suppress the superscript $(L)$.

The PT is a discrete-time version of the modified $z$ transform [56], [57]. At a fixed-time $n=n_{0}$, the PT is the $z$-transform of the signal $x_{n_{0}}[l]=x\left[n_{0}+l L\right]$ derived by subsampling $x\left[n_{0}+n\right]$ by a factor of $L$ :

$$
\mathcal{Z}_{x}\left(n_{0}, z\right)=\sum_{l=-\infty}^{\infty} x_{n_{0}}[l] z^{-l} .
$$

In particular, the PT for $L=1$ evaluated at $n=0$ is the $z$-transform of $x[n], \mathcal{X}(z)=\sum_{n=-\infty}^{\infty} x[n] z^{-n}$ :

$$
\mathcal{Z}_{x}^{(1)}(0, z)=\mathcal{X}(z)
$$

Furthermore, with $z=r e^{j 2 \pi \theta}$, where $r \geq 0$, the PT is seen to be essentially the DTZT of $x[n] r^{-n / L}$ :

$$
\begin{aligned}
\mathcal{Z}_{x}^{(L)}(n, z) & =r^{n / L} \sum_{l=-\infty}^{\infty} x[n+l L] r^{-(n+l L) / L} e^{-j 2 \pi l \theta} \\
& =r^{n / L} \operatorname{DTZT}\left\{x[n] r^{-n / L}\right\} .
\end{aligned}
$$

In the following, we shall discuss important properties and relations of the PT.

2) z-Transform Expression and Region of Convergence: The PT can be expressed in terms of $\mathcal{X}(z)$ as

$$
\mathcal{Z}_{x}(n, z)=\frac{1}{L} z^{n / L} \sum_{m=0}^{L-1} \mathcal{X}\left(z^{1 / L} e^{j 2 \pi(m / L)}\right) e^{j 2 \pi m / L} .
$$

This allows us to determine the region of convergence (ROC) of the PT. If the $z$-transform $\mathcal{X}(z)$ of $x[n]$ converges for $r_{1}<|z|<r_{2}$ with $r_{2}<\infty$, then it follows from (22) that the PT's ROC is $r_{1}^{L}<|z|<r_{2}^{L}$. If $\mathcal{X}(z)$ converges at $\infty$ or at 0 , the same need not be true for $\mathcal{Z}_{x}(n, z)$ due to the factor $z^{n / L}$ in (22). If the PT converges on the unit circle, then the DTZT exists and equals the PT evaluated on the unit circle, i.e.

$$
\left.\mathcal{Z}_{x}^{(L)}(n, z)\right|_{z=e^{j 2 \pi \theta}}=Z_{x}^{(L)}(n, \theta) .
$$

3) Periodicity: The PT satisfies the "quasiperiodicity" relation

$$
\mathcal{Z}_{x}(n+l L, z)=z^{l} \mathcal{Z}_{x}(n, z)
$$

Therefore, it suffices to consider the PT on the fundamental time interval $0 \leq n \leq L-1$. 
4) Integral/Sum Relations: We next summarize important relations involving integrals and/or sums of the PT. The signal $x[n]$ can be recovered from its PT via a contour integral in the $z$-plane:

$$
\frac{1}{2 \pi j} \oint \mathcal{Z}_{x}(n, z) z^{-1} d z=x[n] .
$$

The contour of integration is assumed to be counterclockwise, to enclose the origin, and to lie entirely in the ROC of $\mathcal{Z}_{x}(n, z)$. If the contour of integration is the unit circle, then (23) can be shown to simplify to the DTZT inversion formula (3). We can also recover the signal's $z$-transform from $\mathcal{Z}_{x}(n, z)$ :

$$
\sum_{n=0}^{L-1} \mathcal{Z}_{x}\left(n, z^{L}\right) z^{-n}=\mathcal{X}(z)
$$

This is equivalent to the polyphase decomposition of $\mathcal{X}(z)$ [28] (see Section V).

The PT also preserves inner products and norms

$$
\left\langle\mathcal{Z}_{x}, \mathcal{Z}_{y}\right\rangle=\langle x, y\rangle \text { and }\left\|\mathcal{Z}_{x}\right\|=\|x\|
$$

where the inner product and norm in the PT domain are defined $\mathrm{as}^{3}$

$$
\begin{aligned}
\left\langle\mathcal{Z}_{x}, \mathcal{Z}_{y}\right\rangle & =\frac{1}{2 \pi j} \sum_{n=0}^{L-1} \oint \mathcal{Z}_{x}(n, z) \tilde{\mathcal{Z}}_{y}(n, z) z^{-1} d z \\
\left\|\mathcal{Z}_{x}\right\| & =\sqrt{\left\langle\mathcal{Z}_{x}, \mathcal{Z}_{x}\right\rangle} \\
& =\sqrt{\frac{1}{2 \pi j} \sum_{n=0}^{L-1} \oint \mathcal{Z}_{x}(n, z) \tilde{\mathcal{Z}}_{x}(n, z) z^{-1} d z .}
\end{aligned}
$$

Two further sum/integral relations are

$$
\begin{aligned}
& \sum_{n=0}^{L-1} \mathcal{Z}_{x}(n, z) \tilde{\mathcal{Z}}_{y}(n, z) \\
& \quad=\frac{1}{L} \sum_{m=0}^{L-1} \mathcal{X}\left(z^{1 / L} e^{j 2 \pi m / L}\right) \tilde{\mathcal{Y}}\left(z^{1 / L} e^{j 2 \pi m / L}\right) \\
& \frac{1}{2 \pi j} \oint \mathcal{Z}_{x}(n, z) \tilde{\mathcal{Z}}_{y}(n, z) z^{-1} d z \\
& \quad=\sum_{l=-\infty}^{\infty} x[n+l L] y^{*}[n+l L] .
\end{aligned}
$$

5) Unitarity: The PT is a unitary mapping from $l^{2}(\mathbb{Z})$ onto $l^{2}([0, L-1] \times C)$, which is the space of functions $\mathcal{F}(n, z)$ defined on $[0, L-1] \times C$ (with $C$ the set of complex numbers) and satisfying

$$
\|\mathcal{F}\|=\sqrt{\frac{1}{2 \pi j} \sum_{n=0}^{L-1} \oint \mathcal{F}(n, z) \tilde{\mathcal{F}}(n, z) z^{-1} d z<\infty .}
$$

The PT's unitarity has two important consequences:

- The PT is a one-to-one mapping. In particular, to any $\mathcal{F}(n, z) \in l^{2}([0, L-1] \times C)$, we can find a unique signal

${ }^{3}$ The paraconjugate $\tilde{\mathcal{Z}}_{x}(n, z)$ of $\mathcal{Z}_{x}(n, z)$ is defined as $\tilde{\mathcal{Z}}_{x}(n, z)=$ $\mathcal{Z}_{x}^{*}\left(n, 1 / z^{*}\right)$ [37]. Note that on the unit circle paraconjugation reduces to conjugation, i.e., $\tilde{\mathcal{Z}}_{x}\left(n, e^{2 \pi j \theta}\right)=\mathcal{Z}_{x}^{*}\left(n, e^{2 \pi j \theta}\right)$. $x[n] \in l^{2}(\mathbb{Z})$ such that $\mathcal{Z}_{x}(n, z)=\mathcal{F}(n, z)$. This signal can be obtained according to the inversion formula (23) as $x[n]=\frac{1}{2 \pi j} \oint \mathcal{F}(n, z) z^{-1} d z$.

- The PT preserves inner products and norms [see (24)].

Proof of Unitarity: Analogously to the DTZT case considered in Section II, it can be shown that the PT maps the orthonormal basis for $l^{2}(\mathbb{Z})$ given by $g_{l, m}[n]=\frac{1}{\sqrt{L}} g[n-$ $l L] e^{j 2 \pi(m / L) n}$ with $l \in \mathbb{Z}$ and $m \in[0, L-1]$ (where, again, $g[n]=1$ for $0 \leq n \leq L-1$ and $g[n]=0$ otherwise) onto the orthonormal basis for $l^{2}([0, L-1] \times C)$ given by $\mathcal{Z}_{g_{l, m}}(n, z)=$ $\frac{1}{\sqrt{L}} z^{-l} e^{j 2 \pi(m / L) n} \mathcal{Z}_{g}(n, z)=\frac{1}{\sqrt{L}} z^{-l} e^{j 2 \pi(m / L) n}$.

6) Product of Two PT's: The PT product $\mathcal{Z}_{x}(n, z) \tilde{Z}_{y}(n, z)$ can be expressed as

$$
\mathcal{Z}_{x}(n, z) \tilde{\mathcal{Z}}_{y}(n, z)=\frac{1}{L} \sum_{l=-\infty}^{\infty} \sum_{m=0}^{L-1}\left\langle x, y_{l, m}\right\rangle e^{j 2 \pi(m / L) n} z^{-l}
$$

with $y_{l, m}[n]=y[n-l L] e^{j 2 \pi(m / L) n}$. By inversion of (25), we obtain

$\left\langle x, y_{l, m}\right\rangle=\frac{1}{2 \pi j} \sum_{n=0}^{L-1} \oint \mathcal{Z}_{x}(n, z) \tilde{\mathcal{Z}}_{y}(n, z) e^{-j 2 \pi(m / L) n} z^{l-1} d z$.

7) Time-Limited Signals: If $x[n]$ is zero outside the interval $[0, L-1]$, then

$$
\mathcal{Z}_{x}(n, z)=x[n] \text { for } \quad 0 \leq n \leq L-1
$$

If $x[n]$ is zero outside the interval $[0, K L-1]$, then

$$
\frac{1}{K} \sum_{k=0}^{K-1} \mathcal{Z}_{x}\left(n, z e^{j 2 \pi k / K}\right)=x[n] \quad \text { for } \quad 0 \leq n \leq K L-1
$$

8) Initial Value Theorem: If $x[n]$ is a causal sequence, i.e., $x[n]=0$ for $n<0$, then

$$
\lim _{|z| \rightarrow \infty} \mathcal{Z}_{x}(n, z)=x[n] \text { for } 0 \leq n \leq L-1
$$

9) Reverse PT: We define the reverse PT as the PT with reversed time index [38]:

$$
\mathcal{Z}_{x}^{\prime}(n, z) \triangleq \mathcal{Z}_{x}(-n, z)=\sum_{l=-\infty}^{\infty} x[-n+l L] z^{-l}
$$

We note that $\mathcal{Z}_{x}^{\prime}(n, z)=z^{-1} \mathcal{Z}_{x}(L-n, z)$. The reverse PT will be used in Section V.

10) Signal Transformation Properties, Symmetry Relations, and Transform Pairs: Table IV lists PT relations involving various signal transformations. We note that the ROC of $a \mathcal{Z}_{x}(n, z)+b \mathcal{Z}_{y}(n, z)$ is given by the intersection of the ROC's of $\mathcal{Z}_{x}(n, z)$ and $\mathcal{Z}_{y}(n, z)$. Table $\mathrm{V}$ summarizes symmetry relations, and Table VI provides some basic PT transform pairs. 
TABLE IV

Signal Transformation Properties of the PT

\begin{tabular}{|l|l||l|l|}
\hline Signal & $\mathrm{PT}$ & Signal & $\mathrm{PT}$ \\
\hline$a x[n]+b y[n]$ & $a \mathcal{Z}_{x}(n, z)+b \mathcal{Z}_{y}(n, z)$ & $x[n] * y[n]$ & $\sum_{m=0}^{L-1} \mathcal{Z}_{x}(n-m, z) \mathcal{Z}_{y}(m, z)$ \\
$x[-n]$ & $\mathcal{Z}_{x}\left(-n, \frac{1}{z}\right)$ & $x[n K]$ & $\mathcal{Z}_{x}^{(K L)}(n K, z)$ \\
$x^{*}[n]$ & $\mathcal{Z}_{x}^{*}\left(n, z^{*}\right)$ & $x[n-N]$ & $\mathcal{Z}_{x}(n-N, z)$ \\
$x^{*}[-n]$ & $\mathcal{Z}_{x}^{*}\left(-n, \frac{1}{z^{*}}\right)=\tilde{\mathcal{Z}}_{x}(-n, z)$ & $z_{0}^{-n} x[n]$ & $z_{0}^{-n} \mathcal{Z}_{x}\left(n, z_{0}^{L} z\right)$ \\
$\operatorname{Re}\{x[n]\}$ & $\frac{\mathcal{Z}_{x}(n, z)+\mathcal{Z}_{x}^{*}\left(n, z^{*}\right)}{2}$ & $x[n-l L]$ & $z^{-l} \mathcal{Z}_{x}(n, z)$ \\
$\operatorname{Im}\{x[n]\}$ & $\frac{\mathcal{Z}_{x}(n, z)-\mathcal{Z}_{x}^{*}\left(n, z^{*}\right)}{2 j}$ & $x[n] e^{j 2 \pi \frac{m}{M} n}$ & $e^{j 2 \pi \frac{m}{M} n} \mathcal{Z}_{x}\left(n, z e^{-j 2 \pi \frac{m}{M} L}\right)$ \\
$x_{e}[n]=\frac{x[n]+x^{*}[-n]}{2}$ & $\frac{\mathcal{Z}_{x}(n, z)+\overline{\mathcal{Z}}_{x}(-n, z)}{2}$ & $x[n-l L] e^{j 2 \pi \frac{m}{M} n}$ & $z^{-l} e^{j 2 \pi \frac{m}{M}(n+l L)} \mathcal{Z}_{x}\left(n, z e^{\left.-j 2 \pi \frac{m}{M} L\right)}\right.$ \\
$x_{o}[n]=\frac{x[n]-x^{*}[-n]}{2}$ & $\frac{\mathcal{Z}_{x}(n, z)-\bar{Z}_{x}(-n, z)}{2}$ & $x[n-l L] e^{j 2 \pi \frac{m}{L} n}$ & $z^{-l} e^{j 2 \pi \frac{m}{L} n} \mathcal{Z}_{x}(n, z)$ \\
$x[n] y[n]$ & $\frac{1}{2 \pi j} \oint \mathcal{Z}_{x}\left(n, \frac{z}{v}\right) \mathcal{Z}_{y}(n, v) v^{-1} d v$ & $n x[n]$ & $n \mathcal{Z}_{x}(n, z)-L z \frac{\partial \mathcal{Z}_{x}(n, z)}{\partial z}$ \\
\hline
\end{tabular}

TABLE V

SYMMETRY RELATIONS OF THE PT

\begin{tabular}{|l|l||l|l|}
\hline Signal property & PT property & Signal property & PT property \\
\hline$x[-n]=x[n]$ & $\mathcal{Z}_{x}\left(-n, \frac{1}{z}\right)=\mathcal{Z}_{x}(n, z)$ & $x^{*}[n]=x[n]$ & $\mathcal{Z}_{x}^{*}\left(n, z^{*}\right)=\mathcal{Z}_{x}(n, z)$ \\
$x^{*}[-n]=x[n]$ & $\tilde{\mathcal{Z}}_{x}(-n, z)=\mathcal{Z}_{x}(n, z)$ & $x^{*}[n]=-x[n]$ & $\mathcal{Z}_{x}^{*}\left(n, z^{*}\right)=-\mathcal{Z}_{x}(n, z)$ \\
\hline
\end{tabular}

TABLE VI

PT Transform Pairs. (The PT Expressions Are Valid for $0 \leq n \leq L-1$.)

\begin{tabular}{|l|l||l|l|}
\hline$x[n]$ & $\mathcal{Z}_{x}(n, z)$ & $x[n]$ & $\mathcal{Z}_{x}(n, z)$ \\
\hline$\delta\left[n-n_{0}\right]$ & $\delta\left[n-n_{0}\right], \quad 0 \leq n_{0} \leq L-1$ & $u[n]-u[n-L]$ & 1 \\
$u[n]$ & $\frac{z}{z-1}, \quad|z|>1$ & $a^{n} u[n]$ & $\frac{a^{n} z}{z-a^{L}}, \quad|z|>a^{L}$ \\
\hline
\end{tabular}

\section{DFT FILTER BANKS}

We now discuss the application of the PT to DFT filter banks with critical sampling or integer oversampling. Our discussion essentially follows [37], [41]-[44]. Although some of the results of this section can be extended to more general filter banks [27], [29], we shall restrict our attention to DFT filter banks for the sake of coherence with Section III. (For a detailed discussion of the relations between filter banks and frame theory, see [58]-[62], [27]-[29], and [54].) Based on the equivalence of DFT filter banks and WHF's [28], [59], [63], we shall also reformulate some of the DTZT relations of Section III in terms of the PT.

\section{A. Perfect Reconstruction DFT Filter Banks}

We consider an $M$-channel, perfect reconstruction (PR), DFT filter bank with zero delay, such that $\hat{x}[n]=x[n]$ (see Fig. 1). We assume integer oversampling or critical sampling, i.e., $M=P L$, where $M$ is the number of channels, $L$ is the decimation factor, and $P \in \mathbb{N}$ is the oversampling factor [37]-[44], [58]-[60], [27], [29]. Note that in the critical case,
$P=1$. The transfer functions ( $z$-transforms) of the analysis and synthesis filters are frequency-translated versions of the transfer functions $\mathcal{H}(z)$ and $\mathcal{F}(z)$ of baseband "prototype" analysis and synthesis filters, respectively, i.e.

$$
\mathcal{H}_{k}(z)=\mathcal{H}\left(z W_{M}^{k}\right), \mathcal{F}_{k}(z)=\mathcal{F}\left(z W_{M}^{k}\right), \quad 0 \leq k \leq M-1
$$

with $W_{M}=e^{-j 2 \pi / M}$. Equivalently, the filters' impulse responses are

$$
h_{k}[n]=h[n] W_{M}^{-k n}, f_{k}[n]=f[n] W_{M}^{-k n}, \quad 0 \leq k \leq M-1 .
$$

The reconstructed signal is given by

$$
\hat{x}[n]=\sum_{k=0}^{M-1} \sum_{m=-\infty}^{\infty} v_{k}[m] f_{k}[n-m L]
$$

with the subband signals

$$
v_{k}[m]=\sum_{n=-\infty}^{\infty} x[n] h_{k}[m L-n]=\left\langle x, h_{k, m}^{-*}\right\rangle
$$

where $h_{k, m}^{-}[n]=h_{k}[m L-n](0 \leq k \leq M-1)$, and $*$ stands for complex conjugation. Setting $f_{k, m}[n]=f_{k}[n-m L]$ and 


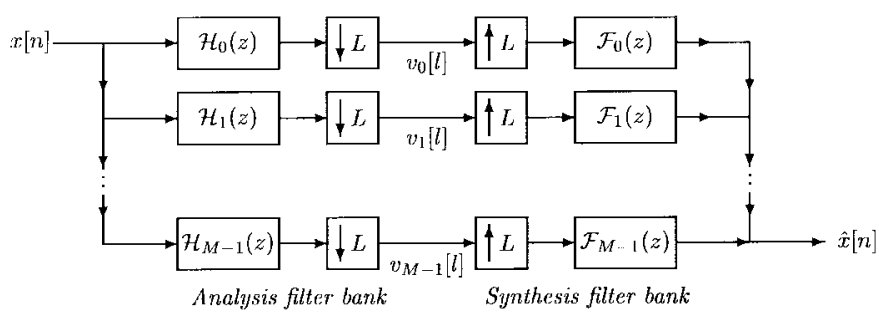

Fig. 1. $\quad M$-channel DFT filter bank with subsampling factor $L$.

using the PR property, we obtain

$$
x[n]=\hat{x}[n]=\sum_{k=0}^{M-1} \sum_{m=-\infty}^{\infty}\left\langle x, h_{k, m}^{-*}\right\rangle f_{k, m}[n] .
$$

Hence, the PR DFT FB provides an expansion of the input signal $x[n]$ into the synthesis functions $f_{k, m}[n]=f[n-$ $m L] W_{M}^{-k \cdot(n-m L)} \quad(0 \leq k \leq M-1,-\infty<m<\infty)$ generated by $f[n]$. The expansion coefficients (= subband signals) $v_{k}[m]=\left\langle x, h_{k, m}^{-*}\right\rangle$ are obtained by projecting $x[n]$ onto the dual functions $h_{k, m}^{-*}[n]=h^{-*}[n-m L] W_{M}^{-k \cdot(n-m L)}$ generated by $h^{-*}[n]=h^{*}[-n]$. Comparing the Gabor synthesis and analysis formulas (7) and (8) with the filter bank synthesis and analysis formulas (27) and (28), respectively, we see that these formulas are fully equivalent if the Gabor analysis and synthesis windows are related to the analysis and synthesis prototype filters, respectively, according to

$$
\gamma[n]=h^{*}[-n], \quad g[n]=f[n] .
$$

In this case, the Gabor coefficients equal the filter bank subband signals up to a phase factor $a_{l, m}=e^{-j 2 \pi(M / N) l m} v_{m}[l]$. Hence, the Gabor expansion (or, equivalently, WHF analysis/synthesis) can be interpreted as a PR DFT filter bank ${ }^{4}$ [59], [60], [28], [63]. Critically sampled DFT FB's correspond to orthogonal or biorthogonal WHF's, whereas oversampled DFT FB's correspond to redundant (overcomplete) WHF's.

\section{B. Application of the Polyphase Transform}

The polyphase decomposition is a well established and important tool in filter bank theory [37]. The polyphase decomposition of the analysis prototype reads

$$
\mathcal{H}(z)=\sum_{n=0}^{L-1} z^{n} \mathcal{Z}_{h}^{\prime}\left(n, z^{L}\right)
$$

where $\mathcal{Z}_{h}^{\prime}(n, z)$ is the reverse PT of $h[n]$ as defined in (26). Similarly, the polyphase decomposition of the synthesis prototype reads ${ }^{5}$

$$
\mathcal{F}(z)=\sum_{n=0}^{L-1} z^{-n} \mathcal{Z}_{f}\left(n, z^{L}\right)
$$

\footnotetext{
${ }^{4}$ There exists, however, the following basic difference: In filter bank theory, one usually specifies the analysis prototype $h[n]$ and computes the corresponding synthesis prototype $f[n]$ for PR. In Gabor expansion (or WHF) theory, on the other hand, the synthesis window $g[n]=f[n]$ is first specified, and then, the corresponding analysis window $\gamma[n]=h^{*}[-n]$ is computed. For reasons of consistency with Section III, we shall here adopt the Gabor expansion viewpoint for filter banks as well.

${ }^{5}$ In the filter bank literature, the polyphase filter transfer functions $\mathcal{Z}_{h}(n, z)$ and $\mathcal{Z}_{f}(n, z)$ are often denoted by $E_{n}(z)$ and $R_{n}(z)$, respectively [37].
}

where $\mathcal{Z}_{f}(n, z)$ is the PT of $f[n]$ as defined in (21). The PT's of the filters $h[n]$ and $f[n]$ are related to the PT's of the Gabor windows $\gamma[n]=h^{*}[-n]$ and $g[n]=f[n]$, respectively, as

$$
\mathcal{Z}_{h}^{\prime}(n, z)=\tilde{\mathcal{Z}}_{\gamma}(n, z), \quad \mathcal{Z}_{f}(n, z)=\mathcal{Z}_{g}(n, z) .
$$

The DTZT relations (13) and (16)-(19) formulated in Section III in a Gabor expansion setting can now be reformulated in terms of the PT, using the analysis and synthesis prototypes $h[n]=\gamma^{*}[-n]$ and $f[n]=g[n]$. This amounts to extending the DTZT relations from the unit circle to the entire $z$-plane.

- In the PT domain, the effect of the frame operator $S$ or inverse frame operator $S^{-1}$ becomes a simple multiplication or division, respectively [28], [60]

$$
\begin{aligned}
\mathcal{Z}_{S_{x}}(n, z) & =\Lambda_{n}(z) \mathcal{Z}_{x}(n, z), \\
\mathcal{Z}_{S^{-1}{ }_{x}}(n, z) & =\frac{\mathcal{Z}_{x}(n, z)}{\Lambda_{n}(z)}
\end{aligned}
$$

with

$$
\begin{gathered}
\Lambda_{n}(z)=L \sum_{p=0}^{P-1} \mathcal{Z}_{f}\left(n, z W_{P}^{p}\right) \tilde{\mathcal{Z}}_{f}\left(n, z W_{P}^{p}\right), \\
0 \leq n \leq L-1
\end{gathered}
$$

where $W_{P}=e^{-j 2 \pi / P}$. Note that the eigenvalues $\lambda_{g}(n, \theta)$ in (13) are $\Lambda_{n}(z)$ evaluated on the unit circle, i.e. $\lambda_{g}(n, \theta)=\left.\Lambda_{n}(z)\right|_{z=e^{j 2 \pi \theta} \text {. }}$

- In the context of DFT filter banks, the frame property is important as well since it guarantees a certain degree of numerical stability (as characterized by the frame bounds $A$ and $B$ of the underlying synthesis WHF) [27], [29]. The frame condition (17) can be reformulated in terms of the PT as

$$
A \leq \Lambda_{n}\left(e^{2 \pi j \theta}\right) \leq B \text { with } 0<A \leq B<\infty
$$

and the frame bounds $A$ and $B$ are the infimum and supremum, respectively, of $\Lambda_{n}\left(e^{2 \pi j \theta}\right)$. In particular, an integer oversampled or critically sampled DFT FB is paraunitary [37] if and only if

$$
\Lambda_{n}(z) \equiv A \quad \text { for } \quad 0 \leq n \leq L-1 .
$$

Note that a DFT filter bank is paraunitary if and only if the underlying WHF is tight [59], [28], [58], [27], [29].

- From a given synthesis prototype $f[n]$ generating a WHF, the analysis prototype $h[n]$ with minimum norm is given by [60]

$$
\mathcal{Z}_{h}^{\prime}(n, z)=\frac{\tilde{\mathcal{Z}}_{f}(n, z)}{\Lambda_{n}(z)}
$$

In the paraunitary (tight) case, there is simply $h[n]=$ $(1 / A) f^{*}[-n]$.

- For a given synthesis prototype $f[n]$ generating a WHF, a "paraunitary" synthesis prototype $f_{T}[n]$ (generating a paraunitary DFT filter bank or, equivalently, a tight WHF) is obtained using

$$
\mathcal{Z}_{f_{T}}(n, z)=\frac{\mathcal{Z}_{f}(n, z)}{\mathcal{C}(z)}
$$

where $\mathcal{C}(z)$ is defined by $\mathcal{C}^{2}(z)=\Lambda_{n}(z)$ and $\tilde{\mathcal{C}}(z)=$ $\mathcal{C}(z)$. 
In the critical case $(P=1)$, we have $\Lambda_{n}(z)=$ $L \mathcal{Z}_{f}(n, z) \tilde{\mathcal{Z}}_{f}(n, z)$, and the above relations simplify accordingly. In particular, (29) reduces to $\mathcal{Z}_{f}(n, z) \tilde{\mathcal{Z}}_{f}(n, z)=$ $A / L$ for $0 \leq n \leq L-1$, which implies $\left|\mathcal{Z}_{f}\left(n, e^{j 2 \pi \theta}\right)\right|^{2} \equiv$ $A / L$. Hence, the polyphase filters $\mathcal{Z}_{f}(n, z)$ are allpass filters, and thus, the design of critically sampled paraunitary DFT FB's reduces to finding an arbitrary set of $L$ allpass filters.

For $P=2$, a paraunitary DFT FB with frame bound $A$ can be constructed by choosing the polyphase filters such that the following power symmetry condition [37] holds:

$$
\begin{gathered}
\mathcal{Z}_{f}(n, z) \tilde{\mathcal{Z}}_{f}(n, z)+\mathcal{Z}_{f}(n,-z) \tilde{\mathcal{Z}}_{f}(n,-z)=\frac{A}{L} \\
\text { for } 0 \leq n \leq L-1 .
\end{gathered}
$$

A detailed treatment of the relation between WHF's and DFT FB's is given in [59], [60], and [28]. We note that the PT can be applied to the more general class of uniform filter banks as well [37]-[39], [58], [59], [27], [29].

\section{The Discrete ZaK TRANSFORM}

For numerical implementations, a ZT version that is discrete in both time and frequency is required. This transform, called discrete Zak transform (DZT), has been introduced in [33] and [34]. The DZT allows an efficient, FFT-based implementation of Gabor analysis and synthesis [12], [33], [34], [16], [10].

1) Definition and Interpretation: If we sample the DTZT $Z_{x}^{(L)}(n, \theta)$ in (1) with respect to frequency $\theta$ at $K$ uniformly spaced sampling points $\theta_{k}=k / K$ and include the normalization factor $1 / \sqrt{K}$, we obtain

$$
\begin{aligned}
Z_{x}^{(L, K)}[n, k] & \triangleq \frac{1}{\sqrt{K}} Z_{x}^{(L)}\left(n, \frac{k}{K}\right) \\
& =\frac{1}{\sqrt{K}} \sum_{l=-\infty}^{\infty} x[n+l L] e^{-j 2 \pi(k / K) l} .
\end{aligned}
$$

From $Z_{x}^{(L, K)}[n, k]$, we can recover only a periodized version of the signal $x[n]$ :

$$
\frac{1}{\sqrt{K}} \sum_{k=0}^{K-1} Z_{x}^{(L, K)}[n, k]=\tilde{x}[n]
$$

with $\widetilde{x}[n]=\sum_{r=-\infty}^{\infty} x[n+r L K]$. The periodized signal $\widetilde{x}[n]$ will contain aliasing in time unless the signal $x[n]$ has finite length $N \leq L K$. Specifically, let us assume that $x[n]$ is zero outside the interval $[0, L K-1]$. In this case, $\tilde{x}[n]=x[n]$ for $n \in[0, L K-1]$. Furthermore, for $n \in[0, L-1], Z_{x}^{(L, K)}[n, k]$ can now be expressed by the finite sum

$$
\begin{aligned}
Z_{x}^{(L, K)}[n, k]=\frac{1}{\sqrt{K}} \sum_{l=0}^{K-1} x[n+l L] e^{-j 2 \pi(k / K) l} \\
\quad 0 \leq n \leq L-1, \quad 0 \leq k \leq K-1 .
\end{aligned}
$$

This is the final definition of the DZT of a finite-length signal $x[n]$ defined for $n \in[0, L K-1]$. Equivalently, we may assume that $x[n]$ is periodic with period $L K$. Thus, the DZT is a linear signal transformation that maps a signal $x[n]$ with length $L K$ (or an $L K$-periodic signal) to a 2 -D function $Z_{x}^{(L, K)}[n, k]$ defined on the fundamental rectangle $(n, k) \in[0, L-1] \times[0, K-1]$. The number of DZT samples is $L K$, which equals the number of signal samples. For a signal of given length (or period) $N$, the DZT parameters $L, K$ must be chosen such that $L K=N$. We shall usually omit the superscript $(L, K)$ in $Z_{x}^{(L, K)}[n, k]$.

The DZT at a fixed time index $n=n_{0}$ is, up to a factor $1 / \sqrt{K}$, the $K$-point discrete Fourier transform (DFT) [55] of the signal $x_{n_{0}}[l]=x\left[n_{0}+l L\right]$ :

$$
\begin{aligned}
Z_{x}\left[n_{0}, k\right] & =\frac{1}{\sqrt{K}} \sum_{l=0}^{K-1} x_{n_{0}}[l] e^{-j 2 \pi(k / K) l} \\
& =\frac{1}{\sqrt{K}} \operatorname{DFT}_{K}\left\{x_{n_{0}}[l]\right\} .
\end{aligned}
$$

In particular, the DZT for $L=1$ and $K=N$ evaluated at $n=0$ is the DFT of $x[n]$ up to a factor:

$$
Z_{x}^{(1, N)}[0, k]=\frac{1}{\sqrt{N}} X[k]
$$

with $X[k]=\operatorname{DFT}_{N}\{x[n]\}=\sum_{n=0}^{N-1} x[n] e^{-j 2 \pi(k / N) n}$.

Thus, in order to compute the DZT for $n \in[0, L-1]$, we have to compute $L$ DFT's of length $K$. This can be done efficiently using an FFT algorithm. Specifically, the DZT is obtained by computing the DFT's (or FFT's) of the columns of the matrix

$$
\boldsymbol{X}=\frac{1}{\sqrt{K}}\left(\begin{array}{cccc}
x[0] & x[1] & \cdots & x[L-1] \\
x[L] & x[L+1] & \cdots & x[2 L-1] \\
x[2 L] & {[2 L+1]} & \cdots & x[3 L-1] \\
\vdots & \vdots & \vdots & \vdots \\
x[N-L] & x[N-L+1] & \cdots & x[N-1]
\end{array}\right) .
$$

2) Frequency-Domain Expression: The DZT can be expressed in terms of the $N$-point DFT (or, equivalently, discrete Fourier series) $X[k]=\sum_{n=0}^{N-1} x[n] e^{-j 2 \pi(k / N) n}$ as

$$
Z_{x}[n, k]=\frac{\sqrt{K}}{N} e^{j 2 \pi n k / N} \sum_{m=0}^{L-1} X[k+m K] e^{j 2 \pi n m / L} .
$$

We next study fundamental properties of the DZT. We assume $x[n] \in \mathcal{P}_{N}$, where $\mathcal{P}_{N}$ is the space of $N$-periodic discrete-time signals with inner product $\langle x, y\rangle=\sum_{n=0}^{N-1} x[n] y^{*}[n]$ and norm $\|x\|=\sqrt{\langle x, x\rangle}=$ $\sqrt{\sum_{n=0}^{N-1}|x[n]|^{2}}$. As before, we assume that the DZT parameters $L, K$ are chosen such that $L K=N$.

3) Periodicity: Let us, for the moment, extend the DZT definition (30) to $n \in \mathbb{Z}$ and $k \in \mathbb{Z}$ (note, however, that $x[n]$ is still assumed $N$-periodic). The extended DZT is quasiperiodic in $n$ and periodic in $k$, i.e.

$$
\begin{aligned}
Z_{x}[n+l L, k] & =e^{j 2 \pi l k / K} Z_{x}[n, k], \\
Z_{x}[n, k+l K] & =Z_{x}[n, k] \quad \text { with } \quad l \in \mathbb{Z} .
\end{aligned}
$$

Therefore, it is indeed sufficient to know the DZT on the fundamental rectangle $(n, k) \in[0, L-1] \times[0, K-1]$. 
4) Sum Relations: Next, we summarize relations involving sums of the DZT. The signal $x[n]$ can be recovered by summing the DZT over the frequency index $k$ :

$$
\frac{1}{\sqrt{K}} \sum_{k=0}^{K-1} Z_{x}[n, k]=x[n], \quad 0 \leq n \leq L-1 .
$$

We can furthermore recover the $N$-point DFT $X[k]$ according to

$$
\sqrt{K} \sum_{n=0}^{L-1} Z_{x}[n, k] e^{-j 2 \pi(n / N) k}=X[k], \quad 0 \leq k \leq K-1 .
$$

Inserting the DZT definition (30), we obtain

$$
X[k]=\sum_{n=0}^{L-1} e^{-j 2 \pi(n / N) k} \sum_{l=0}^{K-1} x[n+l L] e^{-j 2 \pi(l / K) k} .
$$

Here, the $N$-point DFT is expressed in terms of $L$ DFT's of length $K=N / L$. This is the relation underlying the decimation-in-time FFT algorithm [55].

Besides being invertible as seen above, the DZT also preserves inner products and norms:

$$
\left\langle Z_{x}, Z_{y}\right\rangle=\langle x, y\rangle \quad \text { and } \quad\left\|Z_{x}\right\|=\|x\|
$$

where the inner product and norm in the DZT domain are defined as

$$
\begin{aligned}
\left\langle Z_{x}, Z_{y}\right\rangle & =\sum_{n=0}^{L-1} \sum_{k=0}^{K-1} Z_{x}[n, k] Z_{y}^{*}[n, k], \\
\left\|Z_{x}\right\| & =\sqrt{\left\langle Z_{x}, Z_{x}\right\rangle}=\sqrt{\sum_{n=0}^{L-1} \sum_{k=0}^{K-1}\left|Z_{x}[n, k]\right|^{2} .}
\end{aligned}
$$

Two further sum relations are

$$
\begin{aligned}
& \sum_{k=0}^{K-1} Z_{x}[n, k] Z_{y}^{*}[n, k]=\sum_{l=0}^{K-1} x[n+l L] y^{*}[n+l L] \\
& \sum_{n=0}^{L-1} Z_{x}[n, k] Z_{y}^{*}[n, k]=\sum_{m=0}^{L-1} X[k+m K] Y^{*}[k+m K] .
\end{aligned}
$$

5) Unitarity: The DZT is a unitary mapping from $\mathcal{P}_{N}$ onto $\mathcal{A}_{L, K}$, where $\mathcal{A}_{L, K}$ denotes the space of square-summable functions $F[n, k]$ defined for $n \in[0, L-1]$ and $k \in[0, K-1]$ with inner product $\langle F, G\rangle=\sum_{n=0}^{L-1} \sum_{k=0}^{K-1} F[n, k] G^{*}[n, k]$. The unitarity of the DZT can be proved in a similar manner as the unitarity of the DTZT (see Section II). It has two important consequences.

- The DZT is a one-to-one mapping. In particular, to any $F[n, k] \in \mathcal{A}_{L, K}$, we can find a unique signal $x[n] \in \mathcal{P}_{N}$ such that $Z_{x}[n, k]=F[n, k]$. This signal is obtained according to the inversion formula (31) as $x[n]=\frac{1}{\sqrt{K}} \sum_{k=0}^{K-1} F[n, k]$.

- The DZT preserves inner products and norms [see (33)].
6) Product of Two DZT's: The DZT product $Z_{x}[n, k] Z_{y}^{*}$ $[n, k]$ allows the 2-D DFT representation

$$
\begin{aligned}
& Z_{x}[n, k] Z_{y}^{*}[n, k] \\
& \quad=\frac{1}{N} \sum_{l=0}^{K-1} \sum_{m=0}^{L-1}\left\langle x, y_{l, m}\right\rangle e^{j 2 \pi(m / L) n} e^{-j 2 \pi(l / K) k}
\end{aligned}
$$

with $y_{l, m}[n]=y[n-l L] e^{j 2 \pi(m / L) n}$. Conversely

$$
\left\langle x, y_{l, m}\right\rangle=\sum_{n=0}^{L-1} \sum_{k=0}^{K-1} Z_{x}[n, k] Z_{y}^{*}[n, k] e^{-j 2 \pi(m / L) n} e^{j 2 \pi(l / K) k} .
$$

7) Zeros of the DZT: For $L$ odd and $K$ even, the DZT will assume characteristic zeros in the case of signals satisfying certain symmetry properties: If $x[n]=x[N-1-n]$, then $Z_{x}[(L-1) / 2, K / 2]=0$, and if $x[n]=-x[N-1-n]$, then $Z_{x}[(L-1) / 2,0]=0$.

8) Time-Limited Signals: If $x[n]$ has finite time support $[0, L-1]$ inside the fundamental period $[0, N-1]$, i.e., $x[n]=0$ for $L \leq n \leq N-1$, then

$$
Z_{x}[n, k]=\frac{1}{\sqrt{K}} x[n] \text { for } \quad 0 \leq n \leq L-1 .
$$

If $x[n]$ is zero outside the interval $[0, M L-1]$ with $K / M \in \mathbb{Z}$, then

$$
\frac{\sqrt{K}}{M} \sum_{k=0}^{M-1} Z_{x}\left[n, k \frac{K}{M}\right]=x[n] \quad \text { for } \quad 0 \leq n \leq M L-1 .
$$

This formula can be viewed as a subsampled version of (31).

9) Bandlimited Signals: If the DFT $X[k]$ of $x[n]$ is limited to the interval $[0, K-1]$, i.e., $X[k]=0$ for $K \leq k \leq N-1$, then

$$
Z_{x}[n, k]=\frac{\sqrt{K}}{N} e^{j 2 \pi n k / N} X[k] \text { for } \quad 0 \leq k \leq K-1 .
$$

If $X[k]$ is limited to the interval $[0, M K-1]$ with $L / M \in \mathbb{N}$, then

$$
\begin{aligned}
& \frac{L}{M} \sqrt{K} \sum_{r=0}^{M-1} Z_{x}\left[r \frac{L}{M}, k\right] e^{-j 2 \pi r k /(M K)}=X[k] \\
& \quad \text { for } \quad 0 \leq k \leq M K-1 .
\end{aligned}
$$

This is a subsampled version of (32).

10) Signal Transformation Properties, Symmetry Relations, and Transform Pairs: The effect of various signal transformations on the DZT is summarized in Table VII. We note that $x[n] * y[n]=\sum_{m=0}^{N-1} x[n-m] y[m]$ and that all signals are assumed $N$-periodic. Table VIII summarizes symmetry relations of the DZT, and Table IX provides some basic DZT transform pairs.

\section{CyClic Gabor EXPANSIONS}

We now discuss the application of the DZT to the cyclic (or "discrete") Gabor expansion [8], [10], [33], [20]. This version of the Gabor expansion assumes that all signals and windows 
TABLE VII

Signal Transformation Properties of the DZT

\begin{tabular}{|l|l||l|l|}
\hline Signal & DZT & Signal & DZT \\
\hline$a x[n]+b y[n]$ & $a Z_{x}[n, k]+b Z_{y}[n, k]$ & $x_{o}[n]=\frac{x[n]-x^{*}[-n]}{2}$ & $\frac{Z_{x}[n, k]-Z_{x}^{*}[-n, k]}{2}$ \\
$x[-n]$ & $Z_{x}[-n,-k]$ & $x[n] y[n]$ & $\frac{1}{\sqrt{K} \sum_{l=0}^{K-1} Z_{x}[n, l] Z_{y}[n, k-l]}$ \\
$x^{*}[n]$ & $Z_{x}^{*}[n,-k]$ & $x[n] * y[n]$ & $\sqrt{K} \sum_{m=0}^{L-1} Z_{x}[n-m, k] Z_{y}[m, k]$ \\
$x^{*}[-n]$ & $Z_{x}^{*}[-n, k]$ & $x[n-K]$ & $Z_{x}[n-K, k]$ \\
$\operatorname{Rc}\{x[n]\}$ & $\frac{Z_{x}[n, k]+Z_{x}^{*}[n,-k]}{2}$ & $x[n] e^{j 2 \pi m \frac{n}{N}}$ & $e^{j 2 \pi m \frac{n}{N}} Z_{x}[n, k-m]$ \\
$\operatorname{Im}\{x[n]\}$ & $\frac{Z_{x}[n, k]-Z_{x}^{*}[n,-k]}{2 j}$ & $x[n-l L]$ & $e^{-j 2 \pi \frac{k}{K} l} Z_{x}[n, k]$ \\
$x_{c}[n]=\frac{x[n]+x_{x}^{*}[-n]}{2}$ & $\frac{Z_{x}[n, k]+Z_{x}^{*}[-n, k]}{2}$ & $x[n-l L] e^{j 2 \pi m \frac{n}{N}}$ & $e^{j 2 \pi m \frac{n}{N}} e^{-j 2 \pi \frac{l}{K}(k-m)} Z_{x}[n, k-m]$ \\
\hline
\end{tabular}

TABLE VIII

SYMMETRY RELATIONS OF THE DZT

\begin{tabular}{|l|l||l|l|}
\hline Signal property & DZT property & Signal property & DZT property \\
\hline$x[-n]=x[n]$ & $Z_{x}[-n,-k]=Z_{x}[n, k]$ & $x^{*}[n]=x[n]$ & $Z_{x}^{*}[n,-k]=Z_{x}[n, k]$ \\
$x^{*}[-n]=x[n]$ & $Z_{x}^{*}[-n, k]=Z_{x}[n, k]$ & $x^{*}[n]=-x[n]$ & $Z_{x}^{*}[n,-k]=-Z_{x}[n, k]$ \\
\hline
\end{tabular}

TABLE IX

DZT Transform Pairs. (The Signal Expressions Are Valid for $n \in[0, N-1]$, And the DZT Expressions Are Valid for $(n, k) \in[0, L-1] \times[0, K-1]$.)

\begin{tabular}{|l|l||l|l|}
\hline$x[n]$ & $Z_{x}[n, k]$ & $x[n]$ & $Z_{x}[n, k]$ \\
\hline$\delta\left[n-n_{0}\right]$ & $\frac{1}{\sqrt{K}} \delta\left[n-n_{0}\right], \quad 0 \leq n_{0} \leq L-1$ & $e^{j 2 \pi \frac{k_{0}}{N} n}$ & $\frac{1}{\sqrt{K}} e^{j 2 \pi \frac{k_{0}}{N} n} \delta\left[k-k_{0}\right], \quad 0 \leq k_{0} \leq K-1$ \\
1 & $\sqrt{K} \delta[k]$ & $u[n]-u[n-L]$ & $\frac{1}{\sqrt{K}}$ \\
$a^{n}$ & $\frac{1}{\sqrt{K}} a^{n} \frac{a^{N}-1}{a^{L} e^{-j 2 \pi} \frac{k}{K}-1}$ & \\
\hline
\end{tabular}

have finite length $N$ (or, equivalently, they are $N$-periodic). The cyclic Gabor expansion of a signal $x[n] \in \mathcal{P}_{N}$ is given by

$$
x[n]=\sum_{l=0}^{K-1} \sum_{m=0}^{M-1} a_{l, m} g_{l, m}[n]
$$

with $g_{l, m}[n]=g[n-l L] e^{j 2 \pi(m / M) n}$ with a suitable synthesis window $g[n]$ and $K L=N$. The parameters $K$ and $M$ are related to the signal length (period) $N$ and the oversampling factor $P$ as $K M=P N$, i.e., the number $K M$ of Gabor coefficients $a_{l, m}$ is $P$ times the number $N$ of signal samples $x[n]$. With $N=K L$, we get $M=P L$. Using a suitable analysis window $\gamma[n]$, the Gabor coefficients $a_{l, m}$ can be calculated as

$$
a_{l, m}=\left\langle x, \gamma_{l, m}\right\rangle=\sum_{n=0}^{N-1} x[n] \gamma_{l, m}^{*}[n]
$$

with $\gamma_{l, m}[n]=\gamma[n-l L] e^{j 2 \pi(m / M) n}$.

In the following, we discuss the cases of critical sampling or integer oversampling, i.e., $P \in \mathbb{N}$. This restriction is motivated by the fact that the DZT diagonalizes the Gabor frame operator only for $P \in \mathbb{N}$, although the DZT can also be applied to cyclic Gabor expansions with rational oversampling [12], [16], [54].

\section{A. Cyclic Weyl-Heisenberg Frames}

The theory of frames (cf. Section III-A) can also be applied to the cyclic Gabor expansion. For a detailed discussion of cyclic WHF's, see, for example, [10]. A set of functions $g_{l, m}[n]=g[n-l L] e^{j 2 \pi(m / M) n}(0 \leq l \leq K-1,0 \leq m \leq$ $M-1)$ is said to be a WHF for $\mathcal{P}_{N}$ if for all $x[n] \in \mathcal{P}_{N}$

$$
A\|x\|^{2} \leq \sum_{l=0}^{K-1} \sum_{m=0}^{M-1}\left|\left\langle x, g_{l, m}\right\rangle\right|^{2} \leq B\|x\|^{2}
$$

where $\|x\|^{2}=\sum_{n=0}^{N-1}|x[n]|^{2}$. For synthesis window $g[n]$ such that $\left\{g_{l, m}[n]\right\}$ is a WHF, the cyclic Gabor expansion (34), (35) exists for all $x[n] \in \mathcal{P}_{N}$, and an analysis (or dual) window can be derived from $g[n]$ as

$$
\gamma[n]=\left(\boldsymbol{S}^{-1} g\right)[n]
$$

where the frame operator $S$ is defined as $(\boldsymbol{S} x)[n]=$ $\sum_{l=0}^{K-1} \sum_{m=0}^{M-1}\left\langle x, g_{l, m}\right\rangle g_{l, m}[n]$. Note that the frame operator 
corresponds to a matrix of size $N \times N$. Thus, calculation of the dual window necessitates the inversion of an $N \times N$ matrix, which is computationally intensive for large signal lengths $N$. Fast algorithms for the calculation of the dual Gabor window and the Gabor coefficients are discussed in [21], [31], and [64]-[68].

For a tight WHF (where $A=B$ ), we have $S=A \boldsymbol{I}$, where $\boldsymbol{I}$ is the identity operator on $\mathcal{P}_{N}$, and hence, there is simply $\gamma[n]=(1 / A) g[n]$. From a synthesis window $g[n]$ generating a WHF, we can derive a "tight" synthesis window $g_{T}[n]$ (generating a tight WHF) as

$$
g_{T}[n]=\left(S^{-1 / 2} g\right)[n]
$$

where $S^{-1 / 2}$ is the inverse of the positive definite operator (matrix) square root of $S$.

\section{B. Diagonalization of the Gabor Frame Matrix}

In the cases of critical sampling or integer oversampling, i.e., $P \in \mathbb{N}$, an orthogonal and complete set of eigenfunctions (or eigenvectors) of the Gabor frame operator (or matrix) $S$ is given by

$$
u_{n, k}\left[n^{\prime}\right]=v\left[n^{\prime}-n\right] e^{j 2 \pi(k / N)\left(n^{\prime}-n\right)}, \quad 0 \leq n^{\prime} \leq N-1
$$

with $v[n]=\frac{1}{\sqrt{K}} \sum_{l=0}^{K-1} \delta[n-l L]$, where $0 \leq n \leq L-1,0 \leq$ $k \leq K-1$. The DZT is related with these eigenfunctions as

$$
Z_{x}[n, k]=\left\langle x, u_{n, k}\right\rangle
$$

and the eigenvalues of $S$ can be written in terms of the DZT of $g[n]$ as $^{6}$

$$
\lambda_{g}[n, k]=L \sum_{p=0}^{P-1}\left|Z_{g}\left[n, k-p \frac{N}{M}\right]\right|^{2}
$$

(the proof of these relations is similar to that in the Appendix). It again follows that the DZT diagonalizes the Gabor frame matrix $S$ (cf. Section III-B), which has the following consequences [12], [16]:

- In the DZT domain, the effect of the Gabor frame matrix $S$ or the inverse Gabor frame matrix $S^{-1}$ becomes a simple multiplication or division, respectively:

$$
\begin{aligned}
Z_{S_{x}}[n, k] & =\lambda_{g}[n, k] Z_{x}[n, k], \\
Z_{S^{-1}{ }_{x}}[n, k] & =\frac{Z_{x}[n, k]}{\lambda_{g}[n, k]} .
\end{aligned}
$$

- The frame condition (36) can be reformulated in terms of the DZT of $g[n]$ as

$$
A \leq \lambda_{g}[n, k] \leq B \quad \text { with } \quad 0<A \leq B<\infty .
$$

The frame bounds $A$ and $B$ are the minimum and maximum, respectively, of $\lambda_{g}[n, k]$. In particular, $\left\{g_{l, m}[n]\right\}$ is a tight WHF for $\mathcal{P}_{N}$ if and only if $\lambda_{g}[n, k] \equiv A$.

- The Gabor analysis window $\gamma[n]$ can be computed using

$$
Z_{\gamma}[n, k]=\frac{Z_{g}[n, k]}{\lambda_{g}[n, k]}
$$

\footnotetext{
${ }^{6}$ Note that $\lambda_{g}[n, k]$ and $u_{n, k}\left[n^{\prime}\right]$ are ordinary (i.e., not generalized, cf. Section III-B) eigenvalues and eigenfunctions, respectively.
}

and inverting $Z_{\gamma}[n, k]$ according to (31) $\gamma[n]=$ $\frac{1}{\sqrt{K}} \sum_{k=0}^{K-1} Z_{\gamma}[n, k]$.

- From a synthesis window $g[n]$ generating a WHF, a tight window $g_{T}[n]$ (with frame bound $A=1$ ) can be derived using

$$
Z_{g_{T}}[n, k]=\frac{Z_{g}[n, k]}{\sqrt{\lambda_{g}[n, k]}} .
$$

In the case of critical sampling $(P=1)$, the above relations simplify to

$$
\begin{gathered}
A \leq L\left|Z_{g}[n, k]\right|^{2} \leq B, \quad Z_{\gamma}[n, k]=\frac{1}{L Z_{g}^{*}[n, k]}, \\
Z_{g_{T}}[n, k]=\frac{1}{\sqrt{L}} e^{j \arg \left\{Z_{g}[n, k]\right\}} .
\end{gathered}
$$

\section{Simulation RESUlts}

We now present simulation results demonstrating the application of the DZT to the calculation of dual Gabor windows, frame bounds, and tight Gabor windows within the framework of the cyclic Gabor expansion (see Section VII). Fig. 2(a) shows a Gaussian synthesis window $g[n]$ with period $N=$ 8192. The corresponding dual Gabor window $\gamma[n]$ for oversampling factor $P=2$ and grid parameters $L=512, M=$ 1024 is depicted in Fig. 2(b). Straightforward calculation of $\gamma[n]$ according to (37) would require the inversion of the $N \times N$ frame matrix, which is on the order of $N^{3}$ (recall that $N=8192$ ), followed by a matrix-vector multiplication (on the order of $N^{2}$ ). In contrast, the DZT-based method according to (40) requires the calculation of the DZT $(L$ FFT's of length $K=N / L=16$, which is on the order of $\left.L \cdot K \log _{2} K=N \log _{2} K\right), N$ divisions, and DZT inversion according to (31) ( $L$ FFT's of length $K$, which is on the order of $\left.N \log _{2} K\right)$.

Fig. 2(c) shows the eigenvalues of the frame matrix that were calculated using the DZT according to (39). The frame bounds $A$ and $B$ (which characterize important numerical properties of the Gabor expansion and equal the minimum and maximum, respectively, of the eigenvalues) were obtained as $A=57.6, B=105.2$. Finally, Fig. 2(d) shows the tight Gabor synthesis window $g_{T}[n]$ calculated via the DZT according to (41). Note that direct calculation of the tight Gabor window according to (38) would require the calculation of the inverse square root of the $N \times N$ frame matrix.

\section{CONCLUSION}

We considered three different versions of the Zak transform (ZT) for discrete-time signals, emphasizing fundamental properties and relations. We also discussed the important role played by these transforms in the theory of Gabor expansions (Weyl-Heisenberg frames) and in the related field of DFT filter banks. This role is due to the fact that the ZT diagonalizes fundamental operators and matrices in the cases of critical sampling and integer oversampling. We note that the ZT can also be applied to rationally oversampled Weyl-Heisenberg frames [12], [16], [69] and DFT filter banks [60], as well as to a more general class of frame expansions and filter banks 


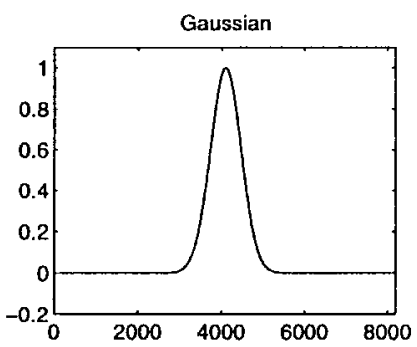

(a)

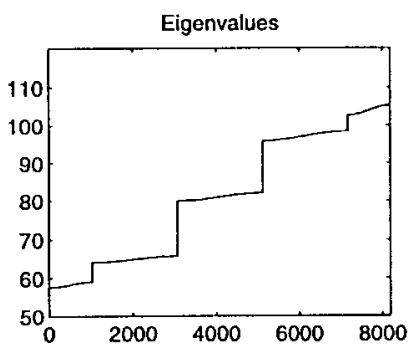

(c)

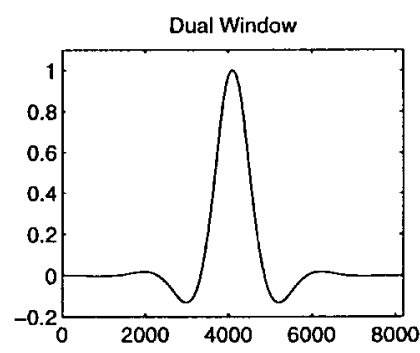

(b)

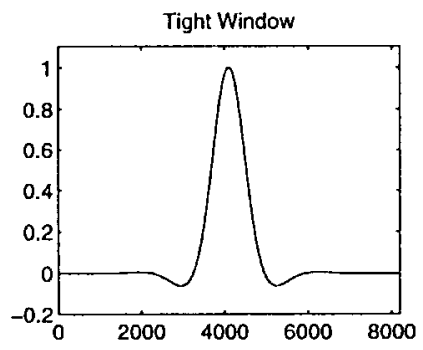

(d)
Fig. 2. DZT-based calculation of the dual (analysis) window, the eigenvalues and frame bounds, and the tight synthesis window. (a) Gaussian synthesis window $g[n]$ with period $N=8192$. (b) Corresponding dual Gabor window $\gamma[n]$ for oversampling factor $P=2$ and grid parameters $L=512, M=1024$. (c) Eigenvalues (sorted in ascending order) of the frame matrix $S$. (d) Tight synthesis window $g_{T}[n]$.

[27], [29], even though it does not perform a diagonalization in these cases.

We have furthermore shown that the extension of the discrete-time ZT to the complex $z$-plane is equivalent to the polyphase representation traditionally used in multirate signal processing and filter bank theory. It is interesting that the essentially equivalent concepts of polyphase representation and ZT have been developed independently in filter bank theory and Gabor expansion theory, respectively.

Finally, simulation results have demonstrated the application of the discrete ZT to the efficient calculation of dual windows, tight windows, and frame bounds for critically sampled and integer oversampled, cyclic Gabor expansions.

\section{APPENDIX \\ EIGENVALUES AND EIGENFUNCTIONS OF THE GABOR FRAME OPERATOR}

We recall from Section III-B that the Gabor frame operator $S$ is defined as

$$
(S x)[n]=\sum_{l=-\infty}^{\infty} \sum_{m=0}^{M-1}\left\langle x, g_{l, m}\right\rangle g_{l, m}[n]
$$

Interchanging the order of summations and using the Poisson sum formula $\sum_{m=0}^{M-1} e^{j 2 \pi(m / M) n}=M \sum_{r=-\infty}^{\infty} \delta[n-r M]$, it is possible to derive the following alternative expression:

$$
(S x)[n]=M \sum_{r=-\infty}^{\infty} x[n-r M] \sum_{l=-\infty}^{\infty} g[n-l L] g^{*}[n-l L-r M]
$$

(a similar expression has been found for the continuoustime Gabor expansion in [12] and [70]). We now restrict our attention to the cases of integer oversampling or critical sampling, i.e., $M=P L$ with $P \in \mathbb{N}$. Inserting $x\left[n^{\prime}\right]=$ $u_{n, \theta}\left[n^{\prime}\right]=\sum_{l=-\infty}^{\infty} \delta\left[n^{\prime}-n-l L\right] e^{j 2 \pi(\theta / L)\left(n^{\prime}-n\right)}$ [see (14)] in (42), we obtain

$$
\begin{aligned}
& \left(\boldsymbol{S} u_{n, \theta}\right)\left[n^{\prime}\right]=P L \sum_{r=-\infty}^{\infty}\left[\sum_{l=-\infty}^{\infty} \delta\left[n^{\prime}-(l+r P) L-n\right]\right. \\
& \left.\cdot e^{j 2 \pi(\theta / L)\left(n^{\prime}-n-r P L\right)}\right] \\
& \cdot \sum_{l_{1}=-\infty}^{\infty} g\left[n^{\prime}-l_{1} L\right] g^{*}\left[n^{\prime}-l_{1} L-r P L\right] \\
& =P L \underbrace{\sum_{l^{\prime}=-\infty}^{\infty} \delta\left[n^{\prime}-n-l^{\prime} L\right] e^{j 2 \pi(\theta / L)\left(n^{\prime}-n\right)}}_{u_{n, \theta}\left[n^{\prime}\right]} \\
& \cdot \sum_{r=-\infty}^{\infty} \sum_{l_{1}=-\infty}^{\infty} e^{-j 2 \pi \theta r P} g\left[n^{\prime}-l_{1} L\right] \\
& \cdot g^{*}\left[n^{\prime}-l_{1} L-r P L\right] \\
& =P L u_{n, \theta}\left[n^{\prime}\right] \sum_{l_{2}=-\infty}^{\infty} \sum_{l_{1}=-\infty}^{\infty} g\left[n^{\prime}-l_{1} L\right] \\
& \cdot g^{*}\left[n^{\prime}-l_{1} L-l_{2} L\right] e^{-j 2 \pi \theta l_{2}} \underbrace{\sum_{r=-\infty}^{\infty} \delta\left[l_{2}-r P\right]}_{\frac{1}{P} \sum_{p=0}^{P-1} e^{j 2 \pi l_{2} p / P}} \\
& =L u_{n, \theta}\left[n^{\prime}\right] \sum_{p=0}^{P-1} \sum_{l_{1}=-\infty}^{\infty} \sum_{l_{2}=-\infty}^{\infty} g\left[n^{\prime}-l_{1} L\right] \\
& \cdot g^{*}\left[n^{\prime}-l_{2} L\right] e^{-j 2 \pi\left(l_{2}-l_{1}\right)(\theta-p / P)} \\
& =u_{n, \theta}\left[n^{\prime}\right] L \sum_{p=0}^{P-1}\left|Z_{g}\left(n, \theta-\frac{p}{P}\right)\right|^{2} \text {. }
\end{aligned}
$$

This shows that $u_{n, \theta}\left[n^{\prime}\right]$ is an eigenfunction of $S$ with eigenvalue $\lambda_{n, \theta}=L \sum_{p=0}^{P-1}\left|Z_{g}\left(n, \theta-\frac{p}{P}\right)\right|^{2}$.

We shall next prove that the $u_{n, \theta}\left[n^{\prime}\right]$ are orthogonal and complete in a generalized sense [53]. According to [53, p. 186], one has to show that for all $x[n] \in l^{2}(\mathbb{Z})$

$$
\begin{aligned}
& \sum_{n=0}^{L-1} \int_{0}^{1}\left|\left\langle x, u_{n, \theta}\right\rangle\right|^{2} d \theta=\|x\|^{2} \text { and } \\
& \sum_{n=0}^{L-1} \int_{0}^{1}\left\langle x, u_{n, \theta}\right\rangle u_{n, \theta}\left[n^{\prime}\right] d \theta=x\left[n^{\prime}\right]
\end{aligned}
$$

where $\left\langle x, u_{n, \theta}\right\rangle=Z_{x}(n, \theta)$. The first equation follows from (5), and the second equation is easily verified to be equivalent to $(3)$.

\section{ACKNOWLEDGMENT}

The authors would like to thank Dr. A. J. E. M. Janssen for valuable suggestions that led to an improvement of this paper. 
They are furthermore grateful to Dr. S. J. L. van Eijndhoven and Prof. H. G. Feichtinger for illuminating discussions and to Prof. W. Mecklenbräuker for drawing their attention to [56] and [57].

\section{REFERENCES}

[1] J. Zak, "Finite translations in solid state physics," Phys. Rev. Lett., vol. 19, pp. 1385-1397, 1967.

[2] A. J. E. M. Janssen, "The Zak transform: A signal transform for sampled time-continuous signals," Philips J. Res., vol. 43, pp. 23-69, 1988.

[3] _ _ "The Zak transform and some counterexamples in timefrequency analysis," IEEE Trans. Inform. Theory, vol. 38, pp. 168-171, Jan. 1992.

[4] H. Bacry, A. Grossmann, and J. Zak, "Proof of completeness of lattice states in the kq representation," Phys. Rev. B, vol. 12, no. 4, pp. 1118-1120, Aug. 1975 .

[5] M. Boon, J. Zak, and J. Zucker, "Rational von Neumann lattices," $J$. Math. Phys. vol. 24, no. 2, pp. 316-323, Feb. 1983.

[6] D. Gabor, "Theory of communication," J. Inst. Elec. Eng., vol. 93, no. 3, pp. 429-457, 1946.

[7] M. J. Bastiaans, "Gabor's expansion of a signal into Gaussian elementary signals," Proc. IEEE, vol. 68, pp. 538-539, Apr. 1980.

[8] J. Wexler and S. Raz, "Discrete Gabor expansions," Signal Processing, vol. 21, pp. 207-220, 1990.

[9] A. J. E. M. Janssen, "Duality and biorthogonality for Weyl-Heisenberg frames," J. Fourier Anal. Applications, vol. 1, no. 4, pp. 403-437, 1995.

[10] _ "Duality and biorthogonality for discrete-time Weyl-Heisenberg frames," Nat. Lab. Rep. 2/94, RWR-518-RE-94001-ak, Philips Nat. Lab. Eindhoven, The Netherlands, 1994.

[11] I. Daubechies, H. Landau, and Z. Landau, "Gabor time-frequency lattices and the Wexler-Raz identity," J. Fourier Anal. Applications, vol. 1, no. 4, pp. 437-478, 1995.

[12] M. Zibulski and Y. Y. Zeevi, "Oversampling in the Gabor scheme," IEEE Trans. Signal Processing, vol. 41, pp. 2679-2687, Aug. 1993.

[13] _ _ "Frame analysis of the discrete Gabor-scheme," IEEE Trans. Signal Processing, vol. 42, pp. 942-945, Apr. 1994.

[14] _ "Analysis of multi-window Gabor-type schemes by frame methods," Tech. Rep. CC Pub. 101, Technion, Haifa, Israel, Apr. 1995.

[15] _ _ "Multi-window Gabor-type transform for signal representation and analysis," SPIE Proc., vol. 2569, Wavelet Applications in Signal and Image Processing III. San Diego, CA: SPIE, July 1995, pp. 116-127.

[16] _ "Discrete multi-window Gabor-type transforms," Tech. Rep., CC Pub. 124, Technion, Haifa, Israel, Nov. 1995.

[17] H. G. Feichtinger and K. Gröchenig, "Gabor wavelets and the Heisenberg group: Gabor expansions and short time Fourier transform from the group theoretical point of view," in Wavelets: A Tutorial in Theory and Applications, C. K. Chui, Ed. New York: Academic, 1992, pp. 297-359.

[18] J. J. Benedetto and D. F. Walnut, "Gabor frames for $L^{2}$ and related spaces," in Wavelets: Mathematics and Applications, J. J. Benedetto and M. W. Frazier, Eds. Boca Raton, FL: CRC, 1993, pp. 97-162.

[19] M. J. Bastiaans, "Gabor's expansion and the Zak transform for continuous-time and discrete-time signals: Critical sampling and rational oversampling," EUT Rep. 95-E-295, Tech. Univ. Eindhoven, Eindhoven, The Netherlands, Dec. 1995.

[20] S. Qian and D. Chen, "Discrete Gabor transform," IEEE Trans. Signal Processing, vol. 41, pp. 2429-2438, July 1993.

[21] R. S. Orr, "The order of computation for finite discrete Gabor transforms," IEEE Trans. Signal Processing, vol. 41, pp. 122-130, Jan. 1993.

[22] R. Tolimieri and R. S. Orr, "Poisson summation, the ambiguity function, and the theory of Weyl-Heisenberg frames," J. Fourier Anal. Applications, vol. 1, no. 3, pp. 233-247, 1995.

[23] J. M. Morris and Y. Lu, "Discrete Gabor expansion of discrete-time signals in $l^{2}(\mathbb{Z})$ via frame theory," Signal Processing, vol. 40, pp. 155-181, 1994.

[24] S. Qian, K. Chen, and S. Li, "Optimal biorthogonal functions for finite discrete-time Gabor expansion," Signal Processing, vol. 27, pp. 177-185, 1992.

[25] A. J. E. M. Janssen, "The Zak transform and sampling theorems for wavelet subspaces," IEEE Trans. Signal Processing, vol. 41, pp. 3360-3364, Dec. 1993

[26] J. W. M. Bergmans and A. J. E. M. Janssen, "Robust data equalization, fractional tap spacing and the Zak transform," Philips J. Res., vol. 42, pp. 351-398, 1987.
[27] H. Bölcskei, F. Hlawatsch, and H. G. Feichtinger, "Frame-theoretic analysis of filter banks," submitted to IEEE Trans. Signal Processing, Feb. 1996.

[28] _ "Equivalence of DFT filter banks and Gabor expansions," SPIE Proc., vol. 2569, part I, Wavelet Applications in Signal and Image Processing III. San Diego, CA: SPIE, July 1995, pp. 128-139.

[29] _ "Frame-theoretic analysis and design of oversampled filter banks," Proc. IEEE ISCAS-96, Atlanta, GA, May 1996, pp. 409-412, vol. 2.

[30] C. Heil, "A discrete Zak transform," Tech. Rep. MTR-89W000128, MITRE Corp., Danvers, MA, 1989.

[31] H. Bölcskei, H. G. Feichtinger, and F. Hlawatsch, "Diagonalizing the Gabor frame operator," Proc. IEEE UK Symp. Applications TimeFrequency Time-Scale Methods, Univ. Warwick, Coventry, U.K., Aug. 1995, pp. 249-255.

[32] J. J. Benedetto, C. Heil, and D. F. Walnut, "Differentiation and the Balian-Low theorem," J. Fourier Anal. Applications, vol. 1, no. 4, pp. 355-403, 1995.

[33] L. Auslander, I. C. Gertner, and R. Tolimieri, "The discrete Zak transform application to time-frequency analysis and synthesis of nonstationary signals," IEEE Trans. Signal Processing, vol. 39, pp. 825-835, Apr. 1991.

[34] Y. Y. Zeevi and I. C. Gertner, "The finite Zak transform: An efficient tool for image representation and analysis," J. Visual Commun. Image Represent., vol. 3, pp. 13-23, Mar. 1992.

[35] R. S. Orr, "Derivation of the finite discrete Gabor transform by periodization and sampling," Signal Processing, vol. 34, pp. 85-97, 1993.

[36] M. G. Bellanger, G. Bonnerot, and M. Coudreuse, "Digital filtering by polyphase network: Application to sample-rate alteration and filter banks," IEEE Trans. Acoust., Speech, Signal Processing, vol. ASSP-24, pp. 109-114, Apr. 1976.

[37] P. P. Vaidyanathan, Multirate Systems and Filter Banks. Englewood Cliffs, NJ: Prentice-Hall, 1993.

[38] M. Vetterli and J. Kovačević, Wavelets and Subband Coding. Englewood Cliffs, NJ: Prentice-Hall, 1995.

[39] A. N. Akansu and R. A. Haddad, Multiresolution Signal Decomposition New York: Academic, 1992.

[40] M. Vetterli, "A theory of multirate filter banks," IEEE Trans. Acoust., Speech, Signal Processing, vol. ASSP-35, pp. 356-372, Mar. 1987.

[41] R. E. Crochiere and L. R. Rabiner, Multirate Digital Signal Processing. Englewood Cliffs, NJ: Prentice-Hall, 1983.

[42] K. Swaminathan and P. P. Vaidyanathan, "Theory and design of uniform DFT, parallel, quadrature mirror filter banks," IEEE Trans. Circuits Syst., vol. CAS-33, pp. 1170-1191, Dec. 1986.

[43] M. J. T. Smith and T. P. Barnwell, III, "A new filter bank theory for time-frequency representation," IEEE Trans. Acoust., Speech, Signal Processing, vol. ASSP-35, pp. 314-327, Mar. 1987.

[44] P. Vary, "On the design of digital filter banks based on a modified principle of polyphase," AEÜ, vol. 33, no. 7/8, pp. 293-300, 1979.

[45] I. Daubechies, Ten Lectures on Wavelets. Philadelphia, PA: SIAM, 1992.

[46] M. R. Portnoff, "Time-frequency representations of digital signals and systems based on short-time Fourier analysis," IEEE Trans. Acoust., Speech, Signal Processing, vol. ASSP-28, pp. 55-69, Feb. 1980.

[47] S. H. Nawab and T. F. Quatieri, "Short-time Fourier transform," in Advanced Topics in Signal Processing, J. S. Lim and A. V. Oppenheim, Eds. Englewood Cliffs, NJ: Prentice-Hall, 1988.

[48] D. F. Stewart, L. C. Potter, and S. C. Ahalt, "Computationally attractive real Gabor transforms," IEEE Trans. Signal Processing, vol. 43, pp. 77-84, Jan. 1995.

[49] I. Daubechies, A. Grossmann, and Y. Meyer, "Painless nonorthogonal expansions," J. Math. Phys. vol. 27, no. 5, pp. 1271-1283, May 1986.

[50] C. E. Heil and D. F. Walnut, "Continuous and discrete wavelet transforms," SIAM Rev., vol. 31, no. 4, pp. 628-666, Dec. 1989.

[51] A. J. E. M. Janssen, "Some frame bound calculations for WeylHeisenberg frames," Indag. Mathem. N. S., vol. 7, no. 2, pp. 165-183, June 17, 1996.

[52] A. W. Naylor and G. R. Sell, Linear Operator Theory in Engineering and Science, 2nd ed. New York: Springer, 1982.

[53] I. M. Gel'fand and G. E. Shilov, Generalized Functions, vol. 3. New York: Academic, 1967

[54] A. J. E. M. Janssen, "The duality condition for Weyl-Heisenberg frames," to appear in Gabor Analysis: Theory, Algorithms, and Applications, H. G. Feichtinger and T. Strohmer, Eds. Boston, MA Birkhäuser, June 1997

[55] A. V. Oppenheim and R. W. Schafer, Discrete-time Signal Processing. Englewood Cliffs, NJ: Prentice-Hall, 1989.

[56] R. H. Barker, "The pulse transfer function and its application to sampling servo systems," Proc. Inst. Elec. Eng., pt. IV, monograph 43, July 1952. 
[57] E. I. Jury, Theory and Application of the Z-Transform Method. New York: Wiley, 1964.

[58] Z. Cvetković and M. Vetterli, "Oversampled filter banks," to appear in IEEE Trans. Signal Processing.

[59] Z. Cvetković, "Oversampled modulated filter banks and tight Gabor frames in $l^{2}(\mathbb{Z})$, , Proc. IEEE ICASSP-95, Detroit, MI, May 1995, pp. $1456-1459$.

[60] H. Bölcskei, F. Hlawatsch, and H. G. Feichtinger, "Oversampled FIR and IIR DFT filter banks and Weyl-Heisenberg frames," Proc. IEEE ICASSP-96, Atlanta, GA, May 1996, vol. 3, pp. 1391-1394.

[61] J. J. Benedetto and S. Li, "The theory of multiresolution analysis frames and applications to filter banks," submitted to ACHA, 1996.

[62] _ "Multiresolution analysis frames with applications," Proc. IEEE ICASSP-93, Minneapolis, MN, 1993, vol. 3, pp. 304-307.

[63] M. Poize, M. Renaudin, and P. Venier, "The Gabor transform as a modulated filter bank system," in Quatorzième Colloque GRETSI, JuanLes-Pins, France, Sept. 1993, pp. 351-354.

[64] S. Qiu and H. G. Feichtinger, "The structure of the Gabor matrix and efficient numerical algorithms for discrete Gabor expansions," in Proc. SPIE, Visual Commun. Image Processing 94, K. Katsaggelos, Ed., 1994, vol. 2308, pp. 1146-1157.

[65] S. Qiu, H. G. Feichtinger, and T. Strohmer, "Inexpensive Gabor decompositions," in Proc. SPIE, Wavelet Applications in Signal and Image Processing II, A. Laine and M. Unser, Eds., 1994, vol. 2303, pp. 286-294.

[66] S. Qiu and H. G. Feichtinger, "Discrete Gabor structures and optimal representations," IEEE Trans. Signal Processing, vol. 43, pp. 2258-2268, Oct. 1995.

[67] N. Polyak, W. A. Pearlman, and Y. Y. Zeevi, "Orthogonalization of circular stationary vector sequences and its application to the Gabor decomposition," IEEE Trans. Signal Processing, vol. 43, pp. 1778-1789, Aug. 1995.
[68] P. Prinz, "Calculating the dual Gabor window for general sampling sets," IEEE Trans. Signal Processing, vol. 44, pp. 2078-2082, Aug. 1996.

[69] A. J. E. M. Janssen, "On rationally oversampled Weyl-Heisenberg frames," Signal Processing, vol. 47, pp. 239-245, 1995.

[70] D. F. Walnut, "Continuity properties of the Gabor frame operator," $J$. Math. Anal. Appl., vol. 165, pp. 479-504, 1992.

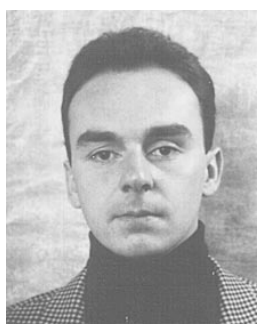

Helmut Bölcskei (S'94) was born in Austria on May 29, 1970. He received the Dipl.-Ing. degree (summa cum laude) from Vienna University of Technology, Austria, in October 1994.

From October to November 1994, he was a Researcher at the Department of Mathematics, University of Vienna, Austria. Since December 1994, he has been a Researcher at the Department of Communications and Radio-Frequency Engineering, Vienna University of Technology. From February 1996 to May 1996, he was a Visiting Researcher in the Applied Mathematics Group at Philips Research Laboratories, Eindhoven, The Netherlands, where he worked on video coding. He is currently pursuing the $\mathrm{Ph} . \mathrm{D}$. degree in electrical engineering. His research interests include filter banks, frame theory, image coding, and time-frequency analysis.

Franz Hlawatsch (M'88), for photograph and biography, please see the February 1997 issue of this TRANSACTIONS. 